\title{
Proceedings of the First International Conference on Food Chemistry \& Technology (FCT-2015)
}

\section{Keynote Abstracts}

\section{Phytonutrients and Antioxidant Activity in Traditional, Native American Edible Plants from Southern California}

\author{
M. Margaret Barth ${ }^{\simeq}$, John Smith and Fred Cox \\ California Baptist University, USA
}

\begin{abstract}
Reports shows the consumption of plant-derived foods such as fruits and vegetables reduces risk of cardiovascular disease, cancer and diabetes due in part to the action of phytonutrients that are capable of neutralizing free radicals and reducing oxidative damage. The objective of this study was to analyze the phytonutrients and other compositional attributes of both fresh and cooked Prickly Pear cactus (Opuntia englemanniis), a Native American edible plant from Southern California. Prickly Pear cactus is a desert plant that has a long history with the San Manuel Band of Serrano Mission Indians and is used for a variety of purposes. Post-harvest handling included sorting for uniformity and quality, and removal of field heat. Samples were prepared and analyzed for moisture, $\mathrm{pH}$ \%Brix, total carotene, total phenolics and flavonoids. The results for the fresh vs. cooked samples were higher for moisture ( $89.0 \%$ vs. 87.0), total carotene ( $3.4 \mathrm{mg} / \mathrm{g}$ DW vs. $3.0 \mathrm{mg} / \mathrm{g}$ DW) and total phenolics (7.3 mg GAE/g DW vs $4.4 \mathrm{mg}$ GAE/g DW). The HPLC chromatograms of both sample types at $330 \mathrm{~nm}$ showed similar decrease of phenolics in the cooked fruit. The $\mathrm{pH}$ and \%Brix were slightly higher in the cooked samples. Overall results showed the cooking method in our study resulted in reducing antioxidant components in the fruit. This study provides helpful information that could be considered for function food and beverage development with novel ingredients for foodservice organizations.
\end{abstract}

\section{Simple and Fast Gas Chromatography-Tandem Mass Spectrometry for the Analysis of Policosanols in Various Vegetable Oils and Green Tea Leaves}

\author{
Mun Yhung Jung ${ }^{-}$ \\ Woosuk University, South Korea
}

\begin{abstract}
A simple and fast gas chromatography-tandem mass spectrometry with a low limit of detection, high specificity, recovery, and precision was successfully established for the policosanol analysis in various food matrixes. This analytical method does not require the sample purification, resulting in the significant reduction of cost, sample preparation time, and labor. The total PC contents in the vegetable oils varies greatly from 3 to $428 \mathrm{mg} / \mathrm{kg}$ oil. Perilla seed oil showed the highest quantity of policosanols. The policosanol contents in perilla seed oil were greatly dependent on the oil extraction method. Green tea leaf was found to be an exceptionally rich plant-base source of policosanols. Young and tender leaves and old and turf leaves of Camellia sinensis were hand-picked in spring and autumn. The total contents of PC in the leaves were in the range of $726-1363 \mathrm{mg} / \mathrm{kg}$ as determined by a GC-MS/MS. The compositions of PC in the leaves were different with harvest season and types. The total contents of PC in commercial green tea leaves were found to be in the range of $857-1435 \mathrm{mg} / \mathrm{kg}$. The infused green tea leaves contained the higher PC than the non-infused green tea product.
\end{abstract}




\title{
Use of Rapid Analytical Methods for Determination of the Quality of Food Products
}

\author{
Romdhane Karoui \\ Artois University, Charles Viollette Institute, France
}

\begin{abstract}
Public interest in food quality and methods of production has increased significantly in recent decades, due in part to changes in eating habits, consumer behavior, and the increased industrialization and globalization of food supply chains. Demand for high levels of quality in food production obviously requires high standards in quality assurance and process control; satisfying this demand in turn requires appropriate analytical tools for food analysis both during and after production. Food systems are mainly composed of water, carbohydrates, proteins, fats, and other constituents that are present at low concentrations such as vitamins, minerals, etc. All these components may contribute to the shape of the spectrum obtained by spectroscopic methods, although, in practice, the major components dominate. Food heterogeneity results in considerable spectral complexity and conventional approaches to the use of spectra for, for example, quantitative predictions of major compounds may not be applied. A major advance in the application of spectral techniques to food analysis and other commodities has been in the application of powerful mathematical techniques known collectively as chemometrics. The potential and ease of application of these rapid techniques coupled with chemometric tools that have found new applications in the field of quality assurance will be covered. The use of specific techniques (front-face fluorescence, near and mid infrared) coupled with chemometric tools for the classification of food samples according to their technological process and/or storage (e.g., milk, cheese, fish, egg, honey, etc.) will also be illustrated.
\end{abstract}

\section{Germination and Graded Polishing of Cereal and Pseudo-Cereal Improves Nutritional and Functional Properties}

\author{
Naofumi Morita ${ }^{1, \bullet, \check{~}}$ and Pham Van Hung ${ }^{2}$ \\ ${ }^{1}$ Toyo College of Food Technology, Japan \\ ${ }^{2}$ Vietnam National University, Vietnam
}

\begin{abstract}
Germination is the start of life activity in a cereal grain: enzymes cleave polymers and various substances, and form bioactive compounds. In this presentation, we examine the germination of cereals and pseudo-cereals. Until now, several germination methods have been developed. The most classical method is to immerse in water, which was undertaken with brown rice. A pre-germinated rice grain developed showing increased amounts of GABA, free amino acids (FAA), dietary fiber, etc. For germinated high-amylose and waxy-wheat, higher dietary fiber, FAA and phenolics were produced. As for buckwheat, germinated buckwheat was tested in natto and miso paste processing, and the grains showed distinctly increase the amounts of GABA: natto (3.3-times) and miso(1.7-times) paste. Immunoblotting using human IgE bound albumin and globulin proteins in soba natto decreased after fermentation for $36 \mathrm{hrs}$. IgE binding allergenic protein in soba miso paste became weak. RiceTartary buckwheat was germinated using moist air in a heating and humidifying method (short time, high temperature). Cereal grains contain important nutritious components in the bran, seed coat and/or aleurone layers; however, when these nutritious fractions are removed by milling and discarded, less of the nutrient-rich parts of grain are eaten. A polished grading method to obtain wheat flour in steps from the outer surface of the grain, differing from conventional whole flour, has been developed. These polished-graded flours show less allergenic fractions. For bread making, these flours are not suitable for white bread, but sour-bread resulted favorably with hemicellulase and some additives. Germinated cereals can be positively recommended in a daily diet.
\end{abstract}

\section{Massi-Care: A New Model to Prevent Food Borne Illness and to Improve Healthy Food Culture}

\author{
Massimo Cecaro \\ Italian Medical Press Association, Italy
}




\begin{abstract}
Food borne illness is a common, public health problem. It has been estimated that 76 million food borne illness cases occur in the United States every year, which means that one in four Americans contracts a food borne illness annually after eating foods. For that reason, prevention of food borne illness is an important goal that should be achieved in an easy and direct way. Indeed, a crucial role is played from mass media, sometimes with imprecise, not accurate, information about food safety. For that reason, correct knowledge of food hygiene and basis for the food's treatment/cooking should be delivered to consumers in a more natural way, using a new, more understandable, model: Massi-Care (Model of Acquire Scientific Specific Information on Care). A specific QR code could be inserted in the food packaging, where the producer would give an "extra service": specifically, food safety matters, and that would involve the expertise of a multi-disciplinary scientific panel (e.g. MD, DVM, biologists, chemists, epidemiologists). The link, given with the above-mentioned QR Code, could be used to match characteristics of a specific consumer with possible pathology and/or nutritional limitations, aiming to give the right answer if the food is ideal or not for a specific category of individuals (e.g. pregnant, individuals with heart conditions, immune compromised patients). Massi-Care could be a new strategy for all the consumers who want to have information about possible implications of the ingredients for the health. That will be delivered in a friendly, easy and direct way.
\end{abstract}

\title{
Speaker Abstracts
}

\section{Lipid Oxidation in Foods: Natural Solutions}

\author{
Poulson Joseph \\ Kalsec, Inc., USA
}

\begin{abstract}
Lipid/fat influences the nutritive composition as well as eating quality in a variety of food systems, including meat, poultry, seafood, snacks, soups, and dressing etc. Oxidative stability is critical to quality, as it would influence the shelf-life of the finished products. To add to the effect, lipid and protein oxidation also inter-influence, more specifically in muscle foods. Various strategies could be employed to delay lipid oxidation in foods, the utilization of antioxidants being a practically adopted one. With the increasing needs for clean label solutions in food industry due to consumer demands, natural solutions for lipid oxidation is highly relevant. There are various factors that could influence the selection and application of natural antioxidants. The session will address this topic with reference to consumer insights, oxidative challenges in various food systems, and specific examples on the attributes of natural antioxidants as well as their application in various food matrices with emphasis on analytical and sensorial approach. The objective of the session would be to deliver a snapshot of natural solutions for enhancing oxidative stability so that food will appear great, taste better, and last longer.
\end{abstract}

\section{Understanding Phenolic Extraction Through the Physical Properties of Wine Grapes from California}

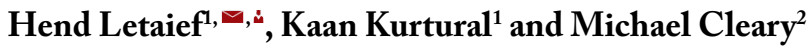 \\ ${ }^{1}$ California State University Fresno, USA \\ ${ }^{2} E$ Eं J Gallo Winery, USA
}

\begin{abstract}
Cell walls are mainly responsible for the integrity and texture of tissues and therefore determine fruit processing. During winemaking, they constitute a diffusion barrier for phenolic compounds. To date, mechanisms responsible for the interaction of anthocyanins and tannins with cell wall material are unclear and hard to predict. Consistent with this, characterizing cell wall physical properties is important for a better understanding of the grape skin extractability potential. Grape berries (Vitis vinifera L., CV Merlot and Cabernet Sauvignon) were harvested at maturation from different regions in California from warm interior valley sites, to cool north coast sites. Grape texture and phenolic composition were determined at commercial harvest and wines were produced from the same lots. Results revealed correlations between berry texture and grape total anthocyanin content. It has been determined that skin elasticity is negatively correlated to an increasing total concentration of anthocyanins in grape skins. Results also showed negative correlations between skin color and skin weight with no varietal effect. Extractability of anthocyanins was more dependent on berry and skin weight than on berry size. Although anthocyanins were higher in northern California grapes, they were less extractible in wines due to higher berry kin elasticity and a higher skin weight.
\end{abstract}




\title{
Salvia officinalis Extracts - A Rich Source of Vitamin E
}

\author{
Radosław Bonikowski ${ }^{1}$, Agnieszka Maciąg1, Magdalena Sikora ${ }^{1}$, Anna Wajs-Bonikowska1, Jozef Kula ${ }^{1, \varpi, ょ, ~ A g n i e s z k a ~}$ \\ Stobiecka $^{1}$, Agnieszka Dobrzyńska-Inger ${ }^{2}$, Dorota Kostrzewa ${ }^{2}$ and Edward Rój ${ }^{2}$ \\ ${ }^{1}$ Institute of General Food Chemistry, Lodz University of Technology, Poland \\ ${ }^{2}$ New Chemical Syntheses Institute, Pulawy, Poland
}

\begin{abstract}
Sage (Salvia officinalis L.), an important herb plant widely used in culinary, pharmacy and cosmetics is the topic of intense research. Chemical solvent extracts (hexane, ethanol) and supercritical $\mathrm{CO}_{2}$ extract of sage were obtained with high yields 24 , 34 and 11\%, respectively, by extraction of dried and finely ground leaves. The presence of tocopherols (vitamin E) has been discovered for the first time in the sage extract by GC-MS methods. Global content of vitamin E in the extracts studied ranged from 1037 to $1778 \mathrm{mg} / 100 \mathrm{~g}$, and more importantly, the most active of these, or $\alpha$-tocopherol constituted 94\%. This work was supported by a grant of The National Centre for Research and Development (Poland), project number: PBS1/A5/18/2012.
\end{abstract}

\section{Detection of GM Soybean Pollen in Mexican Honey by Digital PCR}

\author{
Maricarmen Quirasco ${ }^{1}$, Eric Vides ${ }^{2}$, Carolina Peña ${ }^{1}$, Cindy Estrada ${ }^{1}$, Irma Hernández ${ }^{1}$, Remy Vandame ${ }^{2}$ and Amanda

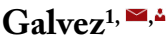 \\ ${ }^{1}$ Universidad Nacional Autonoma de Mexico, Mexico \\ ${ }^{2}$ El Colegio de la Frontera Sur (ECOSUR), Mexico
}

\begin{abstract}
Mexican honey is highly appreciated in conventional and organic markets worldwide. The objective of this work was the detection and quantification of GM pollen sequences in honey. Samples were collected in Mayan communities in Campeche, Mexico in 2013-2014, by ECOSUR, in apiaries surrounded by GM soybean crops potentially visited by honeybees. Pollen DNA was extracted using the guidelines published by the German government, optimized in our lab. Primers and probes used for qPCR (ABI7500) and droplet digital PCR (ddPCR) (BioRad QX200) were public (JRC). Actin was used as endogenous universal target plant gene. Duplex reactions were set for the detection of P-35S/T-nos, and for soy/maize species. GM positives samples were quantitatively analyzed for GM soybean events MON04032/MON89788, also in duplex reactions. In some cases, other pollen, such as cotton and canola's was sought. Even though samples from the rainy season (September) showed the presence of MON04032 pollen, 16 out of 69, results indicated that samples from February 2014 (first harvest) were free from GM pollen. In all samples high quantities of maize pollen were detected. Considering the high diversity of pollen found in multi floral honey, we could show that ddPCR allowed the detection and quantification of GM sequences, even in a very low proportion with respect to total DNA. Such method allowed the analysis of quantities as low as 0.1\% (GM DNA/plant DNA). Findings of GM sequences, not accepted by international organic certification companies, compromises the premium price expected by 14000 Mayan families who produce and export organic honey.
\end{abstract}

\section{Involvement of the Interfacial Layer and Protein Partition on Oxidation of Oil-in- Water Emulsions}

\author{
Claude Genot ${ }^{-}$
}

INRA UR1268 Biopolymères Interactions Assemblages, France

\begin{abstract}
Because of their health benefits, there is a huge interest in dietary formulations enriched with polyunsaturated fatty acids (PUFA)-rich oils. However, these oils are highly sensitive to oxidation, which may cause loss of food sensory and nutritional quality. One clue for controlling lipid oxidation in emulsion-like multiphase systems is to control the local balance between pro- and antioxidants and the access of pro-oxidative factors for the substrates. The interface layer that stabilizes and covers the oil droplets can exhibit a large range of chemical and physical properties depending on the adsorbed surface-active molecules and their organisation at the interface, all determined by ingredients and processes. Controlled design of model oil-in water emulsions allowed to demonstrate that surfactant-stabilized interfaces are more efficient to protect emulsified lipids against
\end{abstract}


oxidation than protein-stabilized interfaces, which was attributed (characterization of Langmuir and Langmuir-Blodgett films) to their greater homogeneity limiting access of reactive species to PUFA. After starting of lipid oxidation, adsorbed proteins become a main target to oxidative attack. In contrast unadsorbed emulsifiers, especially proteins, are able to delay lipid oxidation. The resulting protein alterations, but also their interactions with antioxidants in relation with their partition in the systems should be now better understood.

\title{
Effects of Phenolic Compounds on Myofibrillar Proteins Oxidation Induced by Peroxyl Radicals
}

\author{
Raquel Bridi ${ }^{\circ}$ ¿ , Benjamin Diethelm, Katia Duhalde, Eva Dorta, Eduardo Silva and Camilo López-Alarcón \\ Pontificia Universidad Católica de Chile, Chile
}

\begin{abstract}
In spite of the advances in the last decades regarding the processes involving protein oxidation, the oxidation of meat proteins and its consequences on meat (or related products) quality has been only partially investigated. As a strategy for delaying or inhibiting meat protein oxidation, some investigations have focused on the effect of pure polyphenols or natural extracts on the processes associated with the oxidative modifications of meat proteins. In the present work we studied the progression of protein oxidation of meat extracts induced by AAPH (2,2'-azobis-(2-amidinopropane) hydrochloride) -derived peroxyl radicals, and the protective effect of polyphenols on these processes. The decrease in protein thiols content, together with the production of carbonyl derivatives and hydroperoxides, as well as, protein cross linking and fragmentation, are matter of this study. SDS-PAGE gels studies showed the formation of high molecular weight cross-linked proteins after a four-hour incubation of the proteins with AAPH $(10 \mathrm{mM})$ in phosphate buffer $(\mathrm{pH} 7.4,75 \mathrm{mM})$ at $37^{\circ} \mathrm{C}$. When the same experiments were performed in the presence of polyphenols, such as quercetin, myricetin, caffeic acid and kaempferol $(10$ and $100 \mu \mathrm{M})$ an important protection of the proteins was observed after six hours of incubation with AAPH. Such protection was evidenced by a decrease in the protein cross linking, as well as, by the other parameters evaluated (carbonyls, hydroperoxides, etc.). We conclude that these polyphenols could be considered as good compounds to delay cross linking of meat proteins. However, future investigations are necessary in order to establish the role that other polyphenolic compounds or complex mixtures could play in these processes.
\end{abstract}

\section{Antioxidant, Antimicrobial and Anti-Tyrosinase Activities of Phenolic Extracts from Chilean Berries and Leaves}

\author{
Carol López de Dicastillo, ${ }^{1,2,}$, Gracia Lopez-Carbalho², Virginia Muriel-Galet ${ }^{2}$, Rafael Gavara ${ }^{2}$, Abel Guarda ${ }^{1}$ and \\ María José Galotto ${ }^{1}$ \\ ${ }^{1}$ Universidad de Santiago de Chile (USACH), Chile \\ ${ }^{2}$ Instituto de Agroquímica y Tecnología de Alimentos, LATA-CSIC, Spain
}

\begin{abstract}
Maqui (Aristotelia chilensis) and murta (Ugni molinae Turcz) are native Chilean shrubs that have been used by mapuche people in folk medicine due to their anti-inflamatory, antihemorragic and antimigraine properties. Extracts from maqui and murta fruit and leaves are extremely interesting due to their antioxidant and antimicrobial activities, which are mainly due to the presence of polyphenolic compounds and organic acids. Extraction of such compounds is strongly influenced by several variables, and in this work the effect of the solvent used on the extraction was studied. The solvents assayed were ethanol, water and mixture 1:1 water: ethanol. The antioxidant power of the resulting extracts were measured by three different methods: 2,2-diphenyl-1-picrylhydrazyl (DPPH) and 2, 2-azinobis(3-ethylbenzothiazoline)-6-sulfonate (ABTS•+) radical scavenger activities and FRAP (reducing activity power). Polyphenolic content was also measured through Folin Ciocalteu method. Antimicrobial activity was also analyzed measuring minimum inhibitory concentration (MIC) of these extracts against Escherichia coli and Listeria innocua, Gram-negative and Gram-positive bacteria, respectively, well known because of their importance in food-borne illness. Bacterial cellular damage was studied through SEM microscopy, and viability of cells exposed to most powerful extracts was also analyzed by flow citometry. Inhibiting effect on tyrosinase enzyme was also studied. The highest polyphenolic yield values, antioxidant and antimicrobial activity results were reached with hydroalcoholic mixture. These preliminary results provide the basis for further application of these polyphenol-enriched extracts from maqui and murta fruit and leaves as nutritional, medicinal and cosmetic supplements with potential human health and food conservation benefits.
\end{abstract}




\title{
Role of 18- $\beta$ Glycyrrhetinic Acid on Cisplatin Induced Hepatotoxicity: Plausible Role of ROS, Inflammation and Apoptosis in Wistar Rats
}

\author{
Sarwat Sultana ${ }^{\square,\llcorner}$ and Summya Rashid \\ Jamia Hamdard (Hamdard University), India
}

\begin{abstract}
Cisplatin $(\mathrm{CP})$ is a potent antitumor agent commonly used for the treatment of various malignancies. It has varied undesirable effects such as hepatotoxicity and nephrotoxicity which limits its broad and widespread clinical practice. CP decreases antioxidant and antioxidant enzymes leading to enhanced generation of reactive oxygen species resulting in oxidative stress, inflammation and apoptosis which are implicated in the pathogenesis of CP induced toxicity. Glycyrrhetinic acid (GA), the a glycon of glycyrrhizin, is a natural compound of the roots of Glycyrrbiza glabra. It has been reported to have anti-oxidative, antiinflammatory and anti-cancerous activities. The aim of our study was to check the efficacy of GA on CP induced hepatotoxicity. Rats were subjected to prophylactic oral treatment of GA for 21 days at lower dose of $50 \mathrm{mg} / \mathrm{kg}$ body weight (b.wt) , higher dose of $100 \mathrm{mg} / \mathrm{kg}$ b.wt and single intra peritoneal injection of CP (10 mg/kg b.wt). The probable mechanism of CP induced hepatic toxicity is the generation of free radicals resulting in oxidative stress; activation of inflammation and apoptotic proteins by up regulation of NFkB, COX-2, caspase- 9 and caspase-3 respectively. Prophylactic treatment of GA attenuated hepatic serum toxicity markers like AST, ALT; oxidative stress markers like MDA, PC and XO levels and a significant up regulation of hepatic antioxidant armory like GSH, GPx, GR and CAT activities compared to the control group. Histopathological alterations confirmed biochemical and immune histochemical data. Present results suggest that GA may be a positive modulator in alleviating $\mathrm{CP}$ induced hepatic toxicity.
\end{abstract}

\section{Contamination of Soy for Food and Feed by Intended Use of Herbicides: the Case of Roundup Ready GM Soy}

\author{
Thomas Bøhn \\ GenØk - Center for Biosafety, Norway
}

\begin{abstract}
Food and feed quality is crucial to human and animal health. Quality can be defined as nutritional sufficiency of minerals, vitamins, fats etc but also includes the absence of toxins, whether man-made or from other sources. Surprisingly, almost no data exist in the peer-review literature on herbicide residues in herbicide tolerant genetically modified (GM) plants after close to 20 years on the market. In research recently published by our laboratory we collected soybean samples grown under three typical agricultural conditions: organic, GM, and conventional (but non-GM). The GM soybeans were tolerant to Roundup herbicides, whose active ingredient is glyphosate. We tested these samples for nutrients and elements as well as relevant pesticides including glyphosate and AMPA(itsmainbreakdownproduct).AllindividualsamplesofGM-soycontainedresiduesofbothglyphosateandAMPA, onaverage $9.0 \mathrm{mg} / \mathrm{kg}$. In contrast, no sample from the conventional or the organic soybeans showed residues of these chemicals. This demonstrates that Roundup Ready GM-soybeans sprayed during the growing season take up and accumulate glyphosate and AMPA. Further feeding studies in the model organism Daphnia magna indicate that these residues reduce the quality of the GM soy. In conclusion, the case illustrates that the innovation of improved weed control, enabled by genetic engineering and herbicide tolerant plants (that can be sprayed during the growing season), include unwanted contamination of toxic chemicals from where it was meant to work - on weeds in the environment - to human and animal consumers.
\end{abstract}

\section{Rutin Bioconjugates/Cyclodextrin Supramolecular System with Improved Antioxidant Activity and Enhanced Bioavailability}

\author{
Iulia Pînzaru',ø,ょ, Daniel I. Hădărugă², Francisc Peter ${ }^{2}$ and Cristina A. Dehelean ${ }^{1}$ \\ ${ }^{1}$ Victor Babeş University of Medicine and Pharmacy Timişoara, Romania \\ ${ }^{2}$ Politehnica University of Timişoara, Romania
}

\section{Abstract}

Flavonoids can be widespread in various fruits and vegetables and play a major role in maintaining health status and in 
prevention against many diseases, particularly cardiovascular diseases and some cancers types. Rutin is a glycosylated flavonoid found in fruits such as orange, lemon, lime, in berries, in beverages and also in different plants part and is well known for its pharmacological activities such as antioxidant activity, anti-inflammatory, antitumor, anti-bacterial, anti-viral and antifungal properties. The hydrophilic character of rutin can be a major problem when the compounds meet the lipidic media. The aims of the study were: 1) to obtain bioconjugates of rutin with saturated and unsaturated fatty acids via enzymatic acylation, 2) to encapsulate the bioconjugates in natural cyclodextrins and 3) to evaluate the antioxidant activity (AOA) of the new supramolecular system compared to the native compounds, using the DPPH radical scavenging activity method. The acylation is a very useful process because the esters obtained have a hydrophobic character and can possess: enhanced biological activity, a better bioavailability and can be proper encapsulated in natural cyclodextrins. Evaluation of the antioxidant activity of these systems has been achieve during separate solutions with different concentration of rutin, rutin cyclodextrin complexes, bioconjugates and bioconjugates cyclodextrin complexes. All the compounds tested showed a great antioxidant activity: the esters had an increased activity compared to the pure rutin and the complexes with cyclodextrins preserved the value of AOA of rutin but they presented a better controlled release as compared with this one.

\title{
Australian Native Food Extracts Inhibits Endogenous and Exogenous Advanced Glycation End Products
}

\author{
Permal Deo \\ University of South Australia, Australia
}

\begin{abstract}
Proteins undergo modification on exposure to carbohydrates or lipids to form adduct such as advanced glycation end products (AGEs) and advanced lipoxidation end products (ALEs). AGE/ALEs have been proposed to act via their AGE receptor to activate pro-inflammatory cellular responses resulting in micro- and macrovascular complications. AGEs formation was originally thought to be a consequence of hyperglycemia however increased AGEs levels have been also found in the absence of hyperglycemia and implicated in renal failure, obesity, aging and Alzheimer's disease. Recent studies suggest that diet constitutes an important exogenous source of AGEs and increases the total circulatory AGE levels in the body. Ne-(carboxymethyl) lysine (CML), quantified both in biological and food systems are now used as a marker of global AGE formation. Inhibition of AGE formation derived from antioxidant-rich natural products represents a potential therapeutic strategy in disease complications. Australian native foods only recently have been explored for their health enhancing potentials with high antioxidant activities. Quandong (Santalum acuminatum), bush tomatoes (Solanum centrale) and lemon myrtle (Backhousia citridora) extracts were used to evaluate potential antiglycation activities using endogenous and food derived AGE models. In endogenous model, quandong and bush tomato extracts exhibited different levels of inhibition against fluorescent AGEs, CML levels and protein oxidation biomarkers. In food model, all three food extracts inhibited fluorescent AGEs, CML and fructose amine. Native foods possess potential sources of natural antioxidants that could inhibit endogenous and exogenous AGE formation. The antiglycation properties suggest a possible role of Australian native foods in treatment of AGE related disease complications.
\end{abstract}

\section{Novel Nanocomposite Films Based on Poly (Lactic Acid) and Thymol for Active Packaging}

\author{
Marina Ramos $\varpi^{\varpi, \AA}$, Alfonso Jiménez, Mercedes Peltzer and María del Carmen Garrigós \\ University of Alicante, Spain
}

\begin{abstract}
Novel nano composite films based on poly (lactic acid) (PLA) were prepared by incorporating thymol, as the active additive, and modified montmorillonite (D43B) at two different concentrations. A complete thermal, structural, mechanical and functional characterization of all nano composites was carried out. Thermal stability was not significantly affected by the addition of thymol, but the incorporation of D43B improved mechanical properties and reduced the oxygen transmission rate by the formation of intercalated structures, as suggested by wide angle X-ray scattering patterns and transmission electron microscopy images. The addition of thymol decreased the PLA glass transition temperature, as the result of the polymer plasticization, and led to modification of the elastic modulus and elongation at break. The amount of thymol remaining in these formulations was determined by liquid chromatography (HPLC-UV) and the antioxidant activity by the DPPH spectroscopic method, suggesting that the formulated nano composites could be considered promising antioxidant active packaging material with a biodegradable nature and able to increase foodstuff shelf-life.
\end{abstract}




\title{
Microwave-Assisted Extraction of Phenolic Compounds from Almond Skin By-products (Prunus amygdalus): A Multivariate Analysis
}

\author{
Arantzazu Valdés ${ }^{\varpi, ¿, ~ A n a ~ B e l t r a ́ n, ~ A l f o n s o ~ J i m e ́ n e z ~ a n d ~ M a r i ́ a ~ D e l ~ C a r m e n ~ G a r r i g o ́ s ~}$ \\ University of Alicante, Spain
}

\begin{abstract}
A microwave-assisted extraction (MAE) procedure to isolate phenolic compounds from almond skin by-products was optimized. A three-level, three-factor Box-Behnken design was used to evaluate the effect of almond skin weight, microwave power, and irradiation time on total phenolic content (TPC) and antioxidant activity (DPPH). Almond skin weight was the most important parameter in the studied responses. The best extraction was achieved using $4 \mathrm{~g}, 60 \mathrm{~s}, 100 \mathrm{~W}$, and $60 \mathrm{~mL}$ of $70 \%$ (v/v) ethanol. TPC, antioxidant activity (DPPH, FRAP), and chemical composition (HPLC-DAD-ESI-MS/MS) were determined by using the optimized method from seven different almond cultivars. Successful discrimination was obtained for all cultivars by using multivariate linear discriminant analysis (LDA), suggesting the influence of cultivar type on polyphenol content and antioxidant activity. The results show the potential of almond skin as a natural source of phenolics and the effectiveness of MAE for the reutilization of these by-products.
\end{abstract}

\section{Effect of Processing on the Bioactive Constituents, Antioxidant Potential and Estimated Glycemic Index of Early Maturing Potato Varieties}

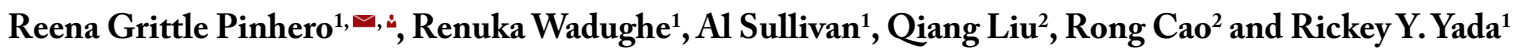 \\ ${ }^{1}$ University of Guelph, Canada \\ ${ }^{2}$ Agriculture and Agri Food Canada, Canada
}

\begin{abstract}
The potato (Solanum tuberosum L.) is one of the most commonly consumed vegetable crops worldwide. The importance of diet to the prevention and management of chronic diseases such as diabetes, obesity and cardiovascular disease is wellrecognized. Increased carbohydrate intake has often been implicated as a prime contributor and, in this light, potatoes can be perceived as being unhealthy due to their carbohydrate rich nature and potentially high glycemic index (GI). However, potatoes are the largest source of dietary antioxidants in the North American diet (after oranges and apples). Furthermore, potatoes contain bioactives such as polyphenols and anthocyanins which act as anticarcinogenic and antimutagenic agents, and resistant starch in potatoes acts as a fibre. The present study evaluated fourteen early maturing varieties of coloured potatoes (white, yellow, red and purple) for bioactive compounds such as polyphenols, resistant and digestible starch, and antioxidant potential after processing. In addition, the glycemic index was estimated (eGI) to evaluate the comparative health benefits of the different varieties. Processing as well as varietal selection affected the content of bioactives, antioxidant potential and eGI. French fingerlings and Purple Fiesta had the lowest eGI irrespective of processing whereas processing lowered eGI in Ciklamen. The total phenolic acid (TPC) content in gallic acid and chlorogenic acid basis were highest in coloured selections F09085 and F10090 with chlorogenic acid being the largest contributor to TPC. Similar trends were observed for antioxidant potential. These results will be discussed in relation to potato as a healthy and affordable vegetable.
\end{abstract}

\section{Cake Flour Chlorination and Alternative Treatments (Review)}

\author{
Hanee M. Al-Dmoor \\ Al-Balqa Applied University, Jordan
}

\begin{abstract}
In order to make an acceptable cake and tenderizers have to be properly balanced. Flour works as a structure builder as it is involved in establishing the crumb structure. Chlorine treatment of soft wheat flour has been widely used since the 1920s. The functional effect of chlorination is on the flour starch, partially de-polymerizing and oxidizing it. Oxidized starch granules are able to swell more rapidly after the starch reaches the gelatinization temperature and the resulting batter system will be sufficiently viscous to support the cake's structure without collapsing. Batter expansion is greater for treated flours, even at the lowest chlorination rate, than for untreated flour. Alternatives to chlorine treatment need to be developed because of the
\end{abstract}


potential carcinogenic effect of chlorination. Developing a practical alternative would start with a study of the mechanism of flour chlorination. Egg albumin contributes strength to cake crumb and improves cake volume. Acceptable cake volume can be achieved by heating but the dryer, stiffer crumb that springs back upon compression is not produced by heated flours. Xanthan gum L-cysteine, and hydrogen peroxide plus peroxidase to heat-treated flour and obtained volumes equivalent to cakes produced using chlorine-treated flour. Ozone can be used as an alternative to chlorine with some benefits. Newly microwave heating treatment of unchlorinated cake flour restores the ability of starch to gelatinize and swell the swollen.

\title{
Model System Points to Strategies for the Fortification of Tea with Iron
}

\author{
Elisa $\mathrm{McGee}^{\curvearrowleft, ~ ¿ ~}$ and Levente Diosady \\ University of Toronto, Canada
}

\begin{abstract}
Iron deficiency is the most widespread nutritional disorder but may be combated though fortification of commonly consumed centrally processed food. Tea is a great candidate because it is the most consumed beverage globally. However, tea contains phenolic compounds that contain ortho-dihydroxyl groups (gallol and catechol moieties) which complex iron. These complexes are not bioavailable and are highly colored. This iron-polyphenol reaction is known to be $\mathrm{pH}$ dependent such that it does not occur, or even reverses, at $\mathrm{pH}$ 1. Therefore, the $\mathrm{pH}$ levels of concern are that of brewed tea and that of the small intestine where the iron and polyphenols are absorbed into the body. To uncover strategies for fortification, a model system using Gallic acid was developed. Tea was characterized based on the amount of polyphenols, $\mathrm{pH}$, and the dependence of these properties on brewing time and tea concentration. Ferrous, ferric, and chelated iron sources were used to investigate the reaction to elucidate potential strategies that may be used in tea fortification. The reaction rate of ferrous iron was found to be $\mathrm{pH}$ dependent, which was not true for ferric iron, as ferrous iron must be first oxidized to form the final phenolic complex. Also, the extent of reaction was found to be much lower for ferric sodium EDTA (a chelated iron source). The results indicate that the use of reducing agents and/or chelating agents will likely have a large effect on slowing the formation of iron-polyphenol complexes in tea.
\end{abstract}

\section{Development of Novel Enrichment Media and Methods of Trace Food Volatiles}

\author{
Zhuomin Zhang \\ Sun Yat-sen University, China
}

\begin{abstract}
Trace food volatiles usually contain potential but important information for food security analysis and quality control. Nowadays, sampling techniques of trace food volatiles still remain one of weak spots in food analysis due to the strong volatility of trace food volatiles from real samples with complicated matrices. Efficient sampling techniques should possess the high extraction capacity and selectivity and can be conveniently coupled with sequent analytical instruments. The core of developing efficient sampling techniques for food volatiles lies in the development of new enrichment media and methods with higher extraction capacity and selectivity. In our previous reports, a series of novel nanoscale enrichment media and methods were developed which mainly focused on the improvement of sampling capacity and selectivity for trace food volatiles. The fabrication formats of these novel enrichment media mainly involved sorbent tube, headspace stir bar and solid-phase micro extraction (SPME) coatings coupling with the consequent analytical instruments. These novel enrichment media and methods have been successfully applied for the selective enrichment and analysis of trace food volatiles from real samples, which is expected to benefit the food security analysis and quality control.
\end{abstract}

\section{Evaluation of Microbial Quality and Antibacterial Activity of Common Herbs in the United Arab Emirates}

Haleama Al Sabbah ־, , Amena Al Zarooni, Rania Dghaim, Usama Alalami and Munawwar Khan

Zayed University, Dubai, UAE

\section{Abstract}

Background: Nowadays, herbs are considered as an alternative to antimicrobial agents that is present in the market. 
Although herbs have curative properties, there are some people who developed intoxications following the use of some herbal plants.

Objective: This study investigated the microbial quality and antibacterial activity of five common herbs in the UAE market including: Parsley, Basil, Sage, Mint, and Thyme.

Methods: In total, 20 herb samples were collected during the period between April-May 2014 from 4 supermarkets and tested, analysed in the university research laboratory. The herbal plant microbial quality was tested for total aerobic bacteria count, yeasts and molds, E. coli, S. aureus, Salmonella and P. aeruginosa. Moreover, antibacterial effect of these herbs on E. coli, S. aureus, Salmonella and P. aeruginosa was tested by using the disk diffusion method.

Results: The microbial analysis of herbs revealed that $50 \%$ of herb samples breached the world health organization (WHO) limit for total aerobic bacteria count, 75\% exceeded the WHO permissible limit for total molds and yeast count, 75\% of herb samples were found contaminated with E. coli and Salmonella, 65\% with Shigella, $10 \%$ exceeded the WHO permissible limit for P. aeruginosa. However, all herb samples were found within the WHO acceptable limit for $S$. aureus. For antibacterial activity, all herb extracts exhibited antibacterial activity against E. coli, S. aureus, Salmonella and P. aeruginosa except for parsley, which was found to have an inhibitory effect on $S$. aureus.

Conclusions: Herbs sold in Emirati market are subject to contamination and may act as a potential hazard to public health. Herbs and other leafy vegetables should be treated directly with certain disinfectant before consumption. It is recommended that the local municipality should impose strict measures to reduce the risk of microbial contamination by applying the Hazard Analysis and Critical Control Point (HACCP). Further studies are needed to assess the effectiveness of these herbs before these are considered as an alternative to modern antimicrobial drugs.

\title{
Inorganic Contaminants and Composition Analysis of Commercial Feed Grade Mineral Compounds Available in Costa Rica
}

\author{
Fabio Granados-Chinchilla ${ }^{1, \varpi,\llcorner,}$, Sugey Prado Mena ${ }^{1}$ and Lisbeth Mata Arias ${ }^{1,2}$ \\ ${ }^{1}$ Centro de Investigación en Nutrición Animal (CINA), Universidad de Costa Rica, Costa Rica \\ ${ }^{2}$ Escuela de Zootecnia, Universidad de Costa Rica, Costa Rica
}

\begin{abstract}
Heavy metals such as arsenic $(\mathrm{As})$, mercury $(\mathrm{Hg})$, lead $(\mathrm{Pb})$ and cadmium $(\mathrm{Cd})$ are potential toxic substances that may incorporate in productive systems in multiple ways including contaminated feedstuff. In this regard, we hypothesize that the main input of heavy metal contamination includes mineral feed ingredients which, in turn, are included in compound feed to meet animals' nutritional requirements. Hence, we offer a comprehensive heavy metal determination on imported feed grade mineral supplement samples $(n=435)$, comprised of 27 different sources including calcium/phosphorus, iron, cobalt, copper, cobalt, manganese, iodide, sulfur, potassium, sodium, selenium and magnesium were collected from eight different local feeding stuff manufactures, mineral and heavy metal as cadmium $(\mathrm{Cd})$, lead $(\mathrm{Pb})$, arsenic $(\mathrm{As})$ and mercury $(\mathrm{Hg})$ analyses were performed using atomic absorption spectroscopy and microwave assisted digestion. Based on this premise, the main goals of the study were to determine mineral and trace mineral content and contrast these values with those advertised by the manufacturers and to determine heavy metal concentrations and compare these levels with the current regulation in commercially available mineral sources which are used in premixes and downstream formulation of compound feeds; a matter which remains undocumented. Our results show that occasionally mineral values for these supplements were lower than those declared. Additionally, several samples contravene, in at least one heavy metal, current regulations; samples in this condition correspond to $0.5(\mathrm{n}=2), 13.8$ $(\mathrm{n}=60), 4.1(\mathrm{n}=18)$ and $2.5 \%(\mathrm{n}=11)$ for $\mathrm{As}, \mathrm{Hg}, \mathrm{Pb}$ and $\mathrm{Cd}$, respectively with mercury as the most frequent mineral to surpass, in the majority of cases, current thresholds. Overall, 21.1\% $(n=92)$ of the samples exhibited concentrations of heavy metals above those stipulated by European guidelines. Meanwhile potassium chloride, $(n=17)$, exhibited the lower overall concentrations of heavy metals. Samples of mineral origin may surpass, in some cases with elevated concentrations, permitted levels of undesirable substances, therefore, a monitoring program for mineral ingredients in our country is recommended.
\end{abstract}

\section{Use of Date Palm (Phoenix dactylifera) to Develop a Medium for Cultivation of Lactobacillus reuteri}

\author{
S. A. Ibrahim ${ }^{1, \varpi, \triangleleft}$ S. O. Aljaloud ${ }^{2}$, A. Ayad ${ }^{1}$, R. Gyawali ${ }^{1}$, D. Gad El-Rab ${ }^{1}$, R. Tahergorabi $^{1}$ and A. Shahbazi ${ }^{1}$ \\ ${ }^{1}$ North Carolina AETT State University, USA \\ ${ }^{2}$ King Saud University, Saudi Arabia
}




\begin{abstract}
Several food processing and agriculture by-products have been tested for the ability to support the growth of lactic acid bacteria and to replace standard laboratory media in order to reduce cost and increase cell mass production. The objective of this study was to investigate the use of date palm (Phoenix dactylifera) as a basic component to develop a medium for cultivation of Lactobacillus reuteri. One hundred gram portions of date were homogenized with $100 \mathrm{~mL}$ distilled water. The mixture was then left at room temperate overnight and filtered using Whatman No. 1 filter. The liquid was centrifuged at $5000 \mathrm{~g}$ for $15 \mathrm{~min}$ at $4^{\circ} \mathrm{C}$ and the clear solution was used as date extract. This extract was mixed with buffer solution (Disodium phosphate $\mathrm{Na}_{2} \mathrm{HPO}_{4}$ $(2 \mathrm{~g})$, ammonium citrate $\mathrm{NH}_{4} \mathrm{C}_{6} \mathrm{H}_{5} \mathrm{O}_{7}(2 \mathrm{~g})$ Sodium acetate $\mathrm{C}_{2} \mathrm{H}_{3} \mathrm{NaO}_{2}(5 \mathrm{~g})$, Calcium chloride $\mathrm{CaCl}_{2}(0.15 \mathrm{~g})$, Dipotassium phosphate $\mathrm{K}_{2} \mathrm{HPO}_{4}(2 \mathrm{~g}), \mathrm{MgSO}_{4} .7 \mathrm{H}_{2} \mathrm{O}(0.2 \mathrm{~g}) \mathrm{MnSO}_{4} \cdot 5 \mathrm{H}_{2} \mathrm{O}(0.05 \mathrm{~g})$, tween $801 \mathrm{~mL}$, and L-Cysteine. HCL ( $\left.1 \mathrm{~g}\right)$ to form a date palm medium (DPM). The final medium was sterilized at $121^{\circ} \mathrm{C}$ for $15 \mathrm{~min}$ and inoculated with active strains of L. reuteri to achieve a final bacterial population of approximately $2.00 \log \mathrm{CFU} / \mathrm{mL}$ Inoculated media were incubated at $37^{\circ} \mathrm{C}$ for 18 $\mathrm{h}$ and bacterial growth was monitored using turbidity (OD at $610 \mathrm{~nm})$, and bacterial population $(\log \mathrm{CFU} / \mathrm{mL})$. Our results showed similar bacterial growth and final cell density in both standard MRS and DPM. These results indicated that DPM could be a suitable medium for the growth of $L$. reuteri and thus could be used as an alternative low cost growth medium.
\end{abstract}

\title{
Considerations in Protein Ingredient Use: the Impact of Modification, Molecular Structure and Interactions
}

\author{
Baraem Ismail $^{\varpi}$ \\ University of Minnesota, USA
}

\begin{abstract}
While proteins have multiple functionality and physiological benefits making them attractive ingredients in many formulations, processing imparts some challenges pertaining to thermal stability, aggregation during storage, Maillard advanced products, and sensory quality, to name a few. Understanding the molecular interactions of various protein ingredients, including isolates and hydrolysates, in systems such as beverages and intermediate moisture foods would aid in choosing the most suitable protein ingredient and processing conditions for a particular application. This presentation will outline the effect of various processing and storage conditions on protein interactions and will highlight the effect of Maillard-induced glycation on molecular configuration as it influences functional properties.
\end{abstract}

\section{Effect of Variety, Treatment and Storage Conditions on the Allergenic Potential of Fresh Fruit}

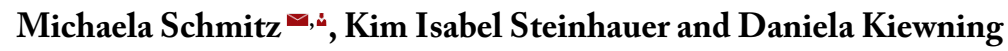

Bonn Rhein Sieg University of Applied Science, Germany

\begin{abstract}
The prevalence of allergies, just as food allergies, is increasing throughout the world. Today up to $70 \%$ of Birch-pollen allergic patients may be food sensitive, especially to apples, peaches and cherries. The parallel appearance of birch and food allergy can be explained by cross-reactive IgE. Consumption of apples and other fruit can provoke several allergic reactions due to the presence of the apple allergen Mal d 1 a protein and similar proteins. The major birch pollen allergen Bet $\mathrm{v} 1$ and the in Central Europe and North America most important apple allergen Mal d 1 and peach allergen Pru p 1 share allergenic epitopes leading to these IgE-cross reactions. Mal d 1 has been identified as a $18 \mathrm{kD}$ protein, present in pulp and peel of apple fruits. Both Bet $\mathrm{v} 1$ and Mal d 1 belong to the so called pathogenesis-related 10 proteins (PRP), a family of proteins that are induced by pathogens or certain environmental stresses. Proteins from peaches or cherries share this similar epitopes too. The objective of the study is to determine the proteins in different cultivars and varieties of fruit leading to allergenic reactions. In the second part of the study, new varieties with minor incidence of allergic symptoms were found or developed.
\end{abstract}




\title{
Nutrition Early in Life and Breast Cancer
}

\author{
Magda Vandeloo ${ }^{1,2,3, \varpi,}$, Liesbeth Bruckers ${ }^{2}$ and Jaak Janssens ${ }^{3}$ \\ ${ }^{1}$ Open University of the Netherlands, The Netherlands \\ ${ }^{2}$ Hasselt University, Belgium \\ ${ }^{3}$ European Cancer Prevention Organisation, Belgium
}

\begin{abstract}
Breast cancer affects one out of 8 women in Western Countries. The main risk factors are early menarche, increased length and weight, late first full-term pregnancy, null parity, late age at menopause, hormonal substitution and high mammographic breast density. Less than 10\% have a hereditary background. Of particular importance is early menarche. Attention to nutrition and lifestyle of children in relation to subsequent breast cancer risk is a relatively recent line of research. Obesity, exposure to estrogens, breast development, and many other risk factors also originate early in life. In fact, breast cancer seems to originate almost entirely during childhood. The breast is most vulnerably at the very onset of development. We studied 1146 healthy girls between birth and the age of 13 for the presence of lifestyle factors that correlate with early puberty and menarche. 46, 7\% period from birth to menarche, show clear evidence that lifestyle factors, including nutrition, do have an effect on both breast development and menarche onset. Management of nutritional factors and lifestyle in children could influence breast cancer risk later in life. Further research should focus on nutrition in children and breast cancer prevention.
\end{abstract}

\section{Bacteriophages Use for Biocontrol of Listeria monocytogenes in Soft Cheese}

\author{
Regina Célia Santos Mendonça ${ }^{\varpi}$,, Jéssica Fernandes Carvalhais, Edimar Aparecida Filomeno Fontes and Maryoris Elisa \\ Soto Lopez
}

Federal University of Viçosa, Brazil

\begin{abstract}
In the dairy industry the importance of L. monocytogenes is due mainly to its ability to develop, even though slowly under refrigeration, to resist to low $\mathrm{pH}$ and salt concentrations above $20 \%$, and to colonize surfaces and form biofilms. Minas cheese (QMF) is a typical Brazilian and widely consumed product, made with pasteurized bovine's milk. This cheese has short shelf life due to its high moisture content, $\mathrm{pH}$ close to neutrality and pronounced handling inherent in manufacturing process. These features constitute a favorable environment to contamination, survival and multiplication by spoilage and pathogenic bacteria. Bacteriophages are specific bacterial viruses. They are mandatorily intracellular parasites due to lack of own metabolism, are host-specific, i.e. able to infect only a specific species or groups in the same species or genus. The objective of this study was evaluating the efficiency of bacteriophage LisIGC in L. monocytogenes biocontrol growing in different matrices. The matrices were pure culture medium, bovine milk added by $\mathrm{NaCl}, \mathrm{CaCl}$ and lactic acid and $\mathrm{QMF}$. The bacteriophage behavior was similar in all conditions, showing that the efficiency in pathogen control was not affected by the tested matrix. In culture medium and modified milk, there was a reduction of 4.0,3.0 and 3.8 logarithmic cycles in pathogen counts after 9, 12 and 12 hours of incubation at $37^{\circ} \mathrm{C}$, respectively. In cheese, the reduction was 3.26 and 1.07 logarithmic cycles after 14 days storage at $15^{\circ} \mathrm{C}$ and $5{ }^{\circ} \mathrm{C}$. It is concluded from this data that the bacteriophage LisIGC was effective in L. monocytogenes growth control not being affected by the raw material or ingredient used for the preparation of QMF.
\end{abstract}

\section{Microencapsulation as an Alternative to Ensure the Viability of Saccharomyces boulardii in Gastrointestinal Tract}

\author{
Hector Eduardo Martinez Flores ${ }^{\square}$ \\ Michoacana University of San Nicolas Hidalgo, Mexico
}

\begin{abstract}
The objective of this work was studied the effect of microencapsulation on the viability of Saccharomyces boulardii in colon of rats fed with freeze-dried yogurt. Two formulations based on freeze-dried yogurt were produced in the first $S$. boulardii was in the form of free cells and the second in microencapsulated form. Viability of yeast in feces and colon of Wistar rats fed with foods was evaluated in normal rats and in rats administered with ampicillin. The evaluation after food intake showed that when the flora of the gastrointestinal tract was affected by ampicillin, $S$. boulardii in foods was detected in colon and feces, when yeast
\end{abstract}


was consumed as free cells or microencapsulated. These results show that $S$. boulardii resisted gastric $\mathrm{pH}$, bile salts and action of digestive enzymes.

\title{
Developing Innovative Healthy Food: Vacuum Impregnation of Apple Slices with Aloe Vera (Aloe barbadensis Mill.) Gels
}

\author{
Antonio Derossi ${ }^{\varpi}$ «, ArcangelaDel Mastro, Teresa De Pilli and Carla Severini \\ University of Foggia, Italy
}

\begin{abstract}
Based on the growing interest toward healthy food, the research of new compounds with biological effects as well as the study of technological solutions for developing functional food has many scientific and practical applications. Recently, scientists discovered the potential healthy effect of Aloe vera gel (Aloe barbadensis Mill.) which shows various properties such as wound healing, anti-fungal, anti-inflammatory, gastro protective, etc. (Choi and Chung, 2003). Among the several components, polysaccharides play an important role in the biological activity of Aloe vera; particularly, acemmannan and glucomannan have immunomodulatory activity (Radha and Laxmipriya, 2015). Vacuum impregnation (VI) is a technique which enables to introduce an external solution into the void phase (pores) of food. VI has been used to accelerate osmotic dehydration and to improve quality attributes of foods (Derossi et al., 2015). The aim of this paper has been to obtain healthy apple slices by vacuum impregnation with Aloevera gel. Experiments were designed trough a central composite design (CCD) of three variables (vacuum level, $\mathrm{p}$, vacuum time, $\mathrm{t} 1$, relaxation time, $\mathrm{t} 2$ ) and five levels of variations. Glucommanan content of samples was measured as healthy index in addition to physical and sensorial attributes. Results showed as VI allowed introducing Aloe vera gels into the pores of apples slice generating a significant increase of their functional properties. Generally, a content of glucomannan ranged between 3 and $15 \mathrm{mg} / 100 \mathrm{~g}$ f.w. was observed. Among the process variables, vacuum level was the most important for increasing the amount of acemmannan into the apple pores.
\end{abstract}

\section{Innovations in Underutilized Cereals and Legume Products to Support Current Trends in the Functional Food Industry}

\author{
Shirani Gamlath \\ Deakin University, Australia
}

\begin{abstract}
Functional Foods are defined as "foods that meet consumer needs for general health and wellbeing, and the prevention and management of compromised health conditions". Health is the future of foods. Functional foods present opportunities for product innovation using underutilized or novel raw materials or modification of existing products to meet the current consumer demand. This presentation summarizes investigations from recent developments in functional products and their functional roles. Emphasis is given to the products containing unexplored cereals and legumes such as sorghum, quinoa, chickpea, and yellow peas. The application of conventional and new technologies in developing these products will also be discussed. These products reported to have proven health benefits to lower glycemic index, improved antioxidant properties and blood lipid profiles, and enhance satiety based on chemical analysis, in vitro and human studies.
\end{abstract}

\section{Functional Food Ingredients for Gut Health}

\author{
Sebnem Tellioglu Harsa \\ Izmir Institute of Technology, Turkey
}

\begin{abstract}
Awareness of the concepts such as healthy living and better nutrition are increasing among the customers worldwide. Functional foods are becoming more important because of increasing healthcare costs, aging population and rising interest in attaining wellness through diet. Functional foods and ingredients targeting gut health constitute one of the largest and fastest growing sectors. Recently there has been a growing appreciation for these beneficial gut microbiota in human and animal health, leading to attempts to manipulate or enhance the microbiota through the use of probiotics, prebiotics and synbiotics. Within
\end{abstract}


this context; isolation, characterization and properties of probiotic lactic acid bacteria and studies on bioactive peptides and proteins based on will be presented.

\title{
Safety Evaluation of Substances Proposed for Use as Food Additives or Nutrient Sources in the European Union (EU)
}

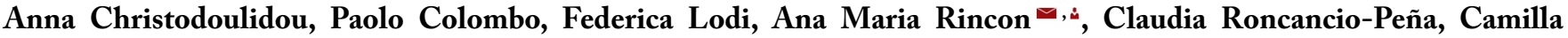 \\ Smeraldi, Stavroula Tasiopoulou and Alexandra Tard
}

European Food Safety Authority (EFSA), Italy

\begin{abstract}
The European Food Safety Authority (EFSA) was created in January 2002 and it is the reference risk assessment body for food safety in the EU. Its main function is to provide independent scientific advice and clear communication on existing and emerging risks. The EFSA Panel on Food Additives and Nutrient Sources Added to Food (ANS) is one of eleven Scientific Panels; which deals with the safety evaluation of food additives, nutrient sources and other substances deliberately added to food, excluding flavorings and enzymes. In the European food safety system, risk assessment is done independently from risk management. A substance shall only be authorized for use as a food additive or nutrient source on the European market if it is demonstrated that it does not represent a risk to human health at the proposed use level. Based on the data provided by the applicant in the dossier and other available information, the ANS Panel issues an independent Scientific Opinion on whether the substance will be of safety concern for human health for the proposed uses, taking into account the relevant toxicological results in animal studies or from human data and exposure estimates. EFSA is also dealing with the re-evaluation of food additives that were already approved before January 2009 and Commission Regulation (EC) No 257/2010 establishes the priorities for their re-evaluation. Since 2009, the re-evaluation of 65 food additives has been finalized. Thirty food additives should be evaluated by 2015 and approximately 200 to be assessed within 2020 with different deadlines.
\end{abstract}

\section{Utility of Microarray Technology as a Detection System for Potential Contaminants}

\author{
Jennifer Catalano ${ }^{1, \bullet, ~ ¿, ~ I a n ~ B e l l a y r ~}{ }^{2}$, Sema Rosinbum$^{2}$ and Subhash Dhawan ${ }^{2}$ \\ ${ }^{1}$ United States International Trade Commission, USA \\ ${ }^{2}$ Food and Drug Administration, USA
}

\begin{abstract}
A microarray consists of a grid of thousands or millions of nucleic acid spots attached to a solid support of a known sequence of adenine, thymine, cytosine, and guanine. Each spot consists of a unique sequence compared to the other spots. The nucleic acid of from an unknown or test experimental sample is isolated and chemically or radioactively labeled. The unknown labeled experimental sample is then combined and hydrogen bonded with the microarray of known nucleic acid spots to generate a quantifiable output signal. This technology can be used as a detection and identification system for samples that contain nucleic acid, whether food or other type of sample. Examples are drawn from years of experience with various samples. The following are discussed, 1) Types of microarrays and comparison with polymerase chain reactions, 2) Appropriate experimental design, 3) Limit of detection and 4) Experimental results with large scale dataset analysis and wide-ranging applications of the technology.
\end{abstract}

\section{Effect of Storage Temperature on Bacterial Growth Rates and Community Structure in Fresh Retail Sushi}

\author{
Sunniva Hoel ${ }^{1,2, \bullet,\llcorner, ~ O l a v ~ V a d s t e i n ~}{ }^{2}$ and Anita Nordeng Jakobsen ${ }^{1}$ \\ ${ }^{1}$ Sør-Trøndelag University College, Norway \\ ${ }^{2}$ Norwegian University of Science and Technology, Norway
}

\begin{abstract}
Retail fresh sushi is gaining popularity in Europe. We have previously assessed the microbiological quality of fresh sushi offered in Norwegian retail stores and revealed a difference between products purchased in stores compared with those collected directly from the factory. The divergence suggests that poor temperature control during distribution and display in stores is a
\end{abstract}


contributor to loss of quality. The aim of the present work was to determine the growth rates of total aerobic plate counts (APC), specific spoilage organisms $\left(\mathrm{H}_{2} \mathrm{~S}\right.$-producing bacteria) and lactic acid bacteria in sushi as a function of storage temperature, and to demonstrate a possible temperature dependent shift in the bacterial community structure using denaturing gradient gel electrophoresis (DGGE) with a nested PCR strategy. Sushi samples $(n=42)$ were collected directly from the production facility and stored at five different temperatures, ranging from 4 to $20{ }^{\circ} \mathrm{C}$. We observed a significant $(\mathrm{p}<0.05)$ increase in the growth rates of all variables at all temperatures $\left(8,12,16\right.$ and $20{ }^{\circ} \mathrm{C}$ ) compared to storage at $4{ }^{\circ} \mathrm{C}$. Storage at $8{ }^{\circ} \mathrm{C}$ increased the growth rate of APC by three times, which clearly demonstrates the importance of strict temperature control of minimally processed ready-to-eat food with a shelf-life of several days. A selection for $\mathrm{H}_{2} \mathrm{~S}$ - producing bacteria at storage temperatures $\geq 12^{\circ} \mathrm{C}$ was observed. The effect of storage temperatures on the selection of the0ptbacterial community structure will be presented in more details.

\title{
Food Safety and Non allergic Food Hypersensitivity Clinical Case Reports from Nutritional Medicine
}

\author{
Claus Muss
}

St. Elisabeth University Bratislava EU, Slovakia

\begin{abstract}
Food safety is in the concern of public health management. Yet a strong controversy about the pathogenesis of adverse health reaction due to food components and chemical additives underpins the general discussion of food intolerance. Uncertainty about relevant test procedure contributes to skepticism and uncertainty in this important field of health care. Food intolerance reactions based on Th1 mediated inflammation and dysfunctional $\mathrm{T}$ reg cell activity are more chronic and less apparent in its presentation and therefore often misdiagnosed. We introduce clinical cases of adverse reactions to food compounds and additives diagnosed by CAST and Lymphocyte transformation test (LTT). Further clinical trials based on such sensitive validated laboratory biomarkers are mandatory to evaluate the true clinical impac
\end{abstract}

\section{Efficacy of Cinnamon and Qysoom Essential Oils, Alone and in Combination, to Retard Lipid Oxidation in Frozen Beef-Burger Model}

\author{
Saddam S. Awaisheh ${ }^{\varpi, 2}$ and Razan J Rahahleh \\ Al-Balqa' Applied University, Jordan
}

\begin{abstract}
Oxidation consequences are detrimental for sensory and shelf-life of meat products. Various antioxidants are used to retard oxidation. The use of natural instead of synthetic antioxidants is increasingly demanded by consumers and legal authorities. Therefore, the objective of this study was to evaluate the effectiveness of essential oils (EOs) of cinnamon (Cinnamomum verum) and qysoom (Achillea arabica) to retard lipid oxidation in frozen beef-burger model. First, EOs antioxidant capacities were evaluated against Butylated-hydroxy-toluene (BHT) on thermally induced oxidation of olive oil $\left(220^{\circ} \mathrm{C} / 2 \mathrm{hrs}\right)$. Then; EOs were added alone or together to the product at concentrations of $0.05 \%$ and $0.1 \%$; while BHT at $0.6 \%$ level served as a standard besides negative control group. Product was stored at $-18{ }^{\circ} \mathrm{C} / 21$ days, and examined for lipid oxidation using thio barbituric acid (TBA) assay, $\mathrm{pH}$ values and sensory criteria at zero day and every week later. Our results showed that the $0.8 \%$ (vol/vol) was the most effective concentration of both EOs to retard oil oxidation, with TBA values 0.28 and 0.14 for cinnamon and qysoom, respectively. The TBA values for negative control and BHT meat samples were 1.316 and 1.124; whereas the cinnamon EO (0.05 and $0.1 \%)$ showed the strongest antioxidant effect with TBA values of 0.725 and 0.868 , respectively; followed by $0.05 \%$ mixture EOs, then qysoom EO, then $0.1 \%$ mixture. Cinnamon $\mathrm{EO}$ at $0.05 \%$ and $0.1 \%$, and $\mathrm{BHT}$ at $0.6 \%$ reduced oxidation rate by $45 \%, 34 \%, 14.6 \%$, respectively, at the end of storage. It might be recommended that the EOs of cinnamon and qysoom could retard the oxidative rancidity of frozen meat products and impart a desirable flavor effect at the same time.
\end{abstract}

\section{A Novel Nonthermal Process for the Concentration of Liquid Foods}

\author{
Fabrice Gascons Viladomat ${ }^{\square, ¿}$, Glenn Pickett, Cédric Grosdemange and Alizé Leblanc
}

EDERNA S.A.S., France 


\title{
Abstract
}

Avoiding product degradation during the concentration stage is difficult when using thermal processes. A novel Engineered Osmosis process developed by EDERNA uses membranes to ensure very high levels of retention of flavor, aroma, and molecular activity while not affecting color. Thermo sensitive molecules are protected from substantial reduction by the absence of heat in the process. No vacuum means that aromatic volatile substances retained. Concentration by the evapEOs ${ }^{\circledR}$ process harnesses the principle of Forward Osmosis. Water is extracted across a particular membrane from the fluid to be concentrated to a patented draw solution. With only 1-2 bars operating pressure on both sides of the membrane, sanitization is easy and greatly reduces fouling. Low temperature membrane technologies treat products well but are significantly limited in concentration capacity (to typically 15-20\% TSS) because of fouling of the pores. When pressure is increased to overcome the resistance, fouling increases and renders cleaning more difficult. Compared to thermal technologies, energy costs are reduced by 30-98\%. An evapEOs system consumes barely $50 \mathrm{kWh} /$ per ton of water extracted. Consumption by thermal evaporation systems is 200 to $800 \mathrm{kWh} /$ ton. Even drying processes can benefit. Using evapEOs upstream of spray drying (consumes up to 1,500 $\mathrm{kWh} / \mathrm{ton}$ ) and lyophilization (up to 5,000 $\mathrm{kWh} /$ ton) provides massive energy savings and increases throughput of existing dryers. Juices, extracts, dairy products and high quality, innovative products have been successfully produced using evapEOs and depending upon the fluid to be concentrated concentration levels can attain up to $65 \%$ TSS.

\section{Comparison of Different Methods for Extraction from Medicinal Plants: Yield, Chemical Composition and Biological Activities}

\author{
Jalloul Bouajila ${ }^{1, \bullet, \_, ~ N e j i a ~ H e r z i ~}{ }^{2}$, Séverine Camy $^{2}$, Mehrez Romdhane ${ }^{3}$ and Jean-Stéphane Condoret ${ }^{2}$ \\ ${ }^{1}$ University of Toulouse, University of Paul-Sabatier, France \\ ${ }^{2}$ Université of Toulouse, France \\ ${ }^{3}$ Research Unit MACS, ENIG, Tunisia
}

\begin{abstract}
Plants remain an inexhaustible source of biologically active substances, among which are the aromatic and medicinal plants that possess a privileged status. The natural products are important in different applications. Especially, the natural antioxidants provide an encouragement for the industry of natural substances, especially food, cosmetic and pharmaceutical sectors. So, the use of supercritical carbon dioxide extraction (SCE) in industry applications can provide several benefits to final products. SCE presented important advantages: extraction is faster, improvement of the yield, a low environmental impact, producing of not degraded compounds, being part of green chemistry and in the optimum process for obtaining extracts with high antioxidant quality. A series of medicinal plant (Eucalyptus cinerea, Eucalyptus camaldulensis, Tetraclinis articulate, Cupressus sempervirens ...) underwent several methods of extraction (solvent extraction, steam distillation, supercritical $\mathrm{CO}_{2}$ with and without co-solvent, extraction with water subcritical) of their secondary metabolites. The performance of different processes have been optimized and compared in terms of yield, chemical composition (GC-MS, HPLC-UV-Vis, LC-MS), duration and the biological activities of extracts. The antioxidant activity was measured using two methods: ABTS •+ and DPPH. Each extraction method has its advantages and can target a chemical family, a physicochemical property or biological activity or other parameters.
\end{abstract}

\section{Synthetic Analogues of Flavonoids as Novel Prototypes of Food Supplements}

\author{
Concettina La Motta ${ }^{1,2, \bullet, ¿, ~ V i t o ~ C o v i e l l o ~}{ }^{1}$, Stefania Sartini ${ }^{1}$, Federico Da Settimo ${ }^{1}$, Serena Del Turco ${ }^{3}$ and Giuseppina \\ Basta $^{3}$ \\ ${ }^{1}$ Department of Pharmacy, University of Pisa, Italy \\ ${ }^{2}$ Interdepartmental Research Center "Nutraceuticals and Food for Health" University of Pisa, Italy \\ ${ }^{3}$ CNR Institute of Clinical Physiology and Fondazione Monasterio, CNR-Regione Toscana, Italy
}

\begin{abstract}
Flavonoids are ubiquitous in fruits and vegetables and show many functional effects, resulting from their ability to modulate key molecular mechanisms related to cardiovascular diseases and some types of cancer. They are present in relatively high amounts in the diet of both European and American population and their intake is highly recommended for preventive health purposes. However, despite endowed with intriguing activities, flavonoids possess significant drawbacks. Actually, their functional effects occur at high, non-physiological concentrations, seldom reached in the circulation, and a clear evidence of the relationship between flavonoids consumption and health benefits still lacks. Moreover, their low solubility and stability, coupled with unfavorable pharmacokinetics properties and the ability to modulate additional and unrelated molecular targets, limit their
\end{abstract}


exploitability either as food supplements or even as drug candidates. Nevertheless, they represent an excellent and logical source of inspiration for medicinal chemists, who may design synthetic analogues to achieve safer and more effective compounds. Prompted by these considerations, we developed a number of synthetic analogues of flavonoids, to obtain novel anti-oxidant, anti-inflammatory compounds as viable agents exploitable in the management of vascular dysfunctions. When tested at a concentration fully consistent with their use in vivo, our derivatives turned out to be more effective than well-known flavonoids in modulating platelet reactivity and regulating key inflammatory events involved in the remodeling of vessel walls. Accordingly, they may represent novel prototypes of food supplements, exploitable to enrich the efficacy of natural flavonoids compounds.

\title{
Generation of Stable Nano-Vesicular Systems Designed for Hyper Anti-oxidative Macromolecular Cargo
}

\author{
Zahur Zee Haque \\ Mississippi State University, USA
}

\begin{abstract}
Oxidative stress results from the intrinsic and extrinsic generation of reactive oxygen species (ROS) and is associated with premature aging and various chronic diseases. Such conditions can be alleviated by consumption of antioxidants as nutraceuticals. As part of our ongoing quest, several food-grade antioxidants have been developed as cargo in nano-vesicular emulsion systems (NVES) to increase their bioavailability. Hydrolysates generated by $\alpha$-chymotrypsin digestion of casein (including $\alpha$ s-casein) exhibited greatly enhanced scavenging abilities against peroxyl and alkoxyl radicals conceivably because of $\pi-\pi$ stacking and/ or $\pi$-cationic residue interactions among aromatic rings. In another study, hydrophilic components extracted from olive leaves (OLE) was found to exhibit marked ROS quenching ability. These cargo materials were subsequently loaded into different NVESs to evaluate their functionality in the emulsion systems. The stable NVESs were formulated by using different types of whey proteins including Edam or Cheddar whey protein concentrate, bovine serum albumin, etc. as primary emulsifiers with/ without low molecular weight surfactants as co-emulsifiers. Ultra-high pressure homogenization was conducted to generate stable nanoemulsion systems with mean particle sizes smaller than $200 \mathrm{~nm}$ and stability up to 2 months. The marked stability and anti-oxidative activities of the NVESs loaded with OLE or curcumin conceivably stemmed from their smaller particle distribution.
\end{abstract}

\section{Changes in Chemical Attributes and Aromatic Profile of Espresso Coffee as Affected by Variables under the Control of the Barista}

\author{
Carla Severini ${ }^{\varpi, ~ ¿, ~ A n t o n i o ~ D e r o s s i, ~ T e r e s a ~ D e ~ P i l l i, ~ I l d e ~ R i c c i ~ a n d ~ M a u r o ~ M a r o n e ~}$ \\ University of Foggia, Italy
}

\begin{abstract}
Espresso coffee quality is affected by several variables some of which are under the control of the barista such as temperature and pressure of water, the grinding grade, the weight of coffee ground, the tamping, etc. (Andueza et al., 2003; Severini et al., 2014). In this paper the changes in quality attributes of espresso coffee (EC) were studied modulating the percolation time, the grinding levels and the tamping. Also, additional tests were performed by using filter holder of 1-cup and 2-cups. Among chemical attributes organic acids, solids, caffeine content and $\mathrm{pH}$ were measured while the global aromatic profile was detected by using an Electronic Nose System (Berna, 2010). Results proved as the major fraction of organic acids, solids and caffeine were extracted in the first 8 seconds of percolation. Also, grinding grade exhibited a great effect on chemical attributes and aroma of EC probably as a consequence of the particle size distribution and the percolation pathways of water through the coffee cake. On the other hand, no differences were observed when the pressure on the surface of coffee cake was modified or when using a filter holder of 1-cup of 2-cup. A multi variate statistical analysis (PCA) enabled to explain more than the $90 \%$ of the variability of the aromatic profile generating an excellent $\mathrm{PC}$ map on which EC samples were precisely separated on the basis of the grinding grade (at constant percolation time) and on percolation fraction (at constant grinding grade).
\end{abstract}




\title{
Application of Isoelectric Solubilization/Precipitation in Recovery of Proteins from Seafood Processing By-products
}

\author{
Reza Tahergorabi \\ North Carolina Agricultural and Technical State University, USA
}

\begin{abstract}
The isoelectric behavior of food proteins has been well characterized. The isoelectric point (pI) of a protein is a $\mathrm{pH}$ at which the protein maintains a zero net electrostatic charge. Therefore, the protein at its $\mathrm{pI}$ exhibits the least solubility; however, as the $\mathrm{pH}$ is changed, the protein-water electrostatic interactions increase; and consequently, the protein becomes water soluble. This fundamental phenomenon has been used to recover functional muscle proteins, particularly fish myofibrillar proteins through a relatively novel method of isoelectric solubilization/precipitation (ISP). It has been reported by numerous popular media that fish stocks are declining and several commercial fisheries are currently over-exploited and will collapse by the mid-century. In general, processing of raw materials for human food products inevitably entails generation of some quantities of processing by-products. However, fish processing typically results in very high amounts of by-products (heads, frames, viscera, and etc.) that are land-filled or ground-and-discarded. When fish are mechanically processed for fillets, the recovery yields are typically $30-40 \%$ of fillets and the by-products account for $60-70 \%$. The $60-70 \%$ of by-products contains highly nutritious fish muscle proteins and fish oil rich in heart-friendly omega- 3 fatty acids. The proteins and oil could be recovered and used subsequently in the development of human food products and dietary supplements. Thus, this presentation focuses on the development of nutraceutical seafood products from ISP-recovered fish proteins by incorporating such ingredients as $\omega$ - 3 oil, dietary fiber, and salt substitute.
\end{abstract}

\section{Binding Fat + Water in Hot Dogs: Capillarity or Emulsion?}

\author{
Wenjie Liu ${ }^{1, \bullet, \AA ~ a n d ~ T y r e ~ C . ~ L a n i e r ~}{ }^{2}$ \\ ${ }^{1}$ General Mills, USA \\ ${ }^{2}$ North Carolina State University, USA
}

\begin{abstract}
Finely comminuted meat products, like frankfurters and luncheon meats, typically contain 20-30\% fat and high water content as well. Classic emulsion theory was initially proposed to explain fat holding ability during cooking, wherein solubilized myofibrillar proteins stabilize the fat/water interface of what was considered to be an emulsion of fat in a protein solution. However, others began to question this simple emulsion concept, however, and placed more emphasis on the concept of the 'entrapment' of fat within the protein gel matrix formed upon cooking. In our study, fat- and nonfat-containing meat gels structurally became more coarse and porous by partial substitution of whey protein isolate for myofibrillar protein, creating not only a weaker texture but also greater cook loss (CL: fat + water) and expressible water (EW). Microstructure examinations revealed a tendency for fat to pool and coalesce during cooking of the more coarse-structured gels. This tendency was unaffected by fat pre-emulsification prior to addition, arguing against emulsion theory (i.e. a strong role of an interfacial protein film in stabilizing fat). More likely the correspondence noted between a homogenous gel structure and low CL and EW seems to be the governing factor in both water- and fat-holding, since fat cannot readily permeate small water-filled hydrophilic pores. Apparently, only when large pores or continuous fissures are present in gel structure can water be released, allowing liquid fat to migrate and coalesce. This changes the paradigm of fat/water-holding in comminuted meat products from meat protein emulsifying ability to gel capillarity (gel structure).
\end{abstract}

\section{Antibacterial Activity Produced by Enterococcus spp. Isolated from an Artisanal Mexican Dairy Product, Cotija Cheese}

\author{
Maricarmen Quirasco $^{1, \bullet, \varkappa}$, C. Eduardo Serrano ${ }^{1}$, Myrna Olvera $^{1}$, Estela Delgado ${ }^{1}$, Carolina Peña ${ }^{1}$, Guillermo Mendoza $^{2}$ \\ and Israel García ${ }^{1}$
}

${ }^{1}$ Facultad de Quimica, Universidad Nacional Autónoma de México, México

${ }^{2}$ Facultad de Medicina, Universidad Nacional Autónoma de México, México 


\title{
Abstract
}

Several Enterococci were isolated from Cotija cheese, which is a Mexican farmhouse-made product prepared from whole raw milk. No starter cultures or any thermal step are used in its manufacturing process. However, a ripening stage of three months is required, as established by the cheesemaker's tradition, for its commercialization. KAA agar was used in order to isolate seven Enterococcus strains, from which two were outstanding for their extracellular lytic activity against Staphylococcus aureus, and were identified by $16 \mathrm{~S}$ rDNA sequencing as Enterococcus faecalis and Enterococcus faecium, respectively. They also showed bacteriolytic activity against other pathogenic bacteria, such as Salmonella enterica Typhimurium, Yersinia enterocolitica, Listeria monocytogenes and Pseudomonas aeruginosa, as demonstrated by agar diffusion tests. By zymography it was shown that they produced proteins with bacteriolytic activity with molecular masses of 95 and $81 \mathrm{kDa}$, respectively. Protein identification by LC/ESI-MS/MS showed that they correspond to different enzymes with peptidoglycan hydrolase (PGH) activity of $\mathrm{N}$-acetylglucosaminidase. Two of them were considered as putative enzymes and had not been studied before. Reducing agents and denaturing treatments demonstrated no protein aggregation and had no effect on lytic activity. Concentrated protein preparations from both strains showed lytic activity on S. aureus growth in liquid culture, as well. Considering that the PGHs studied are produced by lactic bacteria isolated from a dairy product and their spectrum of bacterial inhibition, these enzymes have a potential use in food preservation.

\section{Impact of Soyasaponin I on TLR2 and TLR4 Induced Inflammation in the MUTZ-3- Cell Model}

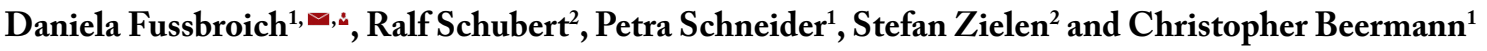 \\ ${ }^{1}$ University of Applied Sciences, Germany \\ ${ }^{2}$ Goethe-University, Germany
}

\begin{abstract}
Previous studies have demonstrated that soyasaponin $(\mathrm{SoSa})$ possesses anti-inflammatory properties in lipopolysaccharide (LPS)-stimulated immune cells by influencing the immune sensing of toll-like receptor (TLR) 4. The aim of this study was to investigate the immune modulatory effect of SoSa I on TLR2- and TLR4-induced inflammation within the human monocytic MUTZ-3-cell model. MUTZ-3 cells were stimulated with gram-negative (Escherichia coli) or gram-positive (Staphylococcus aureus) bacteria or bacterial pathogen-associated molecular patterns (PAMPs) such as LPS or peptidoglycans (PGN) alone or in combination with SoSa I. Cell morphology was characterized by raster scanning and light microscopy. Cytokine production (IL-1 $\beta$, IL-6 and TNF- $\alpha$, IP-10, RANTES and IL-8) was measured by cytometric bead array and the expression of surface markers was assessed by flow cytometry. MUTZ-3 cells revealed a cell maturation-like alteration in morphology and increased expression of CD80, CD86, TLR2 and TLR4 after stimulation with either gram-negative and gram-positive bacteria or bacterial PAMPs. Hereby, SoSa I suppressed pro-inflammatory cytokine and chemokine secretions in a dose-dependent manner regardless of TLR2 or TLR4 stimulation. SoSa I inhibited more efficiently E. coli and S. aureus induced inflammation compared to LPS and PGN. Additionally, SoSa I reduced the expression of CD86 in PGN- or LPS-stimulated cells.

This study demonstrated that the anti-inflammatory capacity of SoSa I is based on influencing both monocytic TLR2 and TLR4. However, the down-regulation of inflammation by SoSa I seems to involve further receptor-ligand recognitions and associated cellular downstream cellular signaling pathways.
\end{abstract}

\section{Proximate Analysis, Functional Properties and Amino Acids Composition of Citrullus lanatus Seed (Melon) Protein Isolate}

\author{
Idiat Bolajoko Ogunyinka ${ }^{\varpi}$, Foluso Oluwagbemiga Osunsanmi, Abidemi Paul Kappo and Andrew Richard Opoku \\ University of Zululand, South Africa
}

\begin{abstract}
Protein energy malnutrition (PEM) is one of the leading causes of death in the developing world, especially among children. Animal proteins are an excellent source of nutritional protein but are relatively unaffordable in poor countries. It has therefore become necessary to seek alternative cheap sources of protein of plant origin. We report on partial chemical analysis of the seeds of Citrullus lanatus, a widely grown type of wild melon traditionally used as a source of oil for human consumption in Africa. We demonstrate for the first time, that the seeds are unusually rich in protein $(63 \%$ by weight) as well as in minerals and essential amino acids, and possess water and oil absorption properties that make them suitable for various kinds of food processing. These attributes make the seeds attractive candidates as a more diverse food source than in the past
\end{abstract}




\title{
Evaluation of Foods on Obesity and Atopic Dermatitis Using Animal Models
}

\author{
Na-Hyung Kim ${ }^{\square}$ \\ Wonkwang University, South Korea
}

\begin{abstract}
Obesity is a growing public health problem and has steadily increased in recent years. For the prevention and treatment of obesity, many foods and raw materials have been concerned and developed. Green pepper (Capsicum annuum L.), a natural product available in many countries, was used by Mexican, Indian more than 2000 years ago and is considered to be a food additive, with healthful application. Capsicum annuum L. includes many components including soluble amino acids, small organic acids, insoluble fatty acids, amino acids, trigonelline, C4-substituted pyridine, choline, cinnamic derivatives, and capsaicin or several related compounds called capsaicinoids. According to observation on anti-obesity effects of green pepper using experimental animals, mice in green pepper juice-administered groups with a high-fat diet gained significantly less weight and represented a significant decrease in serum triglycerides, total cholesterol, low density lipoproteins, and alanine amino transferase compared to mice given only a high-fat diet. Systolic/diastolic blood pressure, heart rate, and blood glucose levels in mice administered green pepper juice were similar to those in mice in the control group. Abdominal fat volume, which was quantified by using $4.7 \mathrm{~T}$ magnetic resonance imaging, in mice of green pepper juice-administered groups with a high-fat diet tended to decrease compared to the fat volume of mice administered only a high-fat diet.
\end{abstract}

\section{Assessment of Indigenous Raw Cow Milk and Selected Milk Products in Ibadan, Nigeria: Bacterial Contaminants from the Environment}

\author{
Olusoji I. Adeyosoye ${ }^{1, \bullet,\llcorner}$ and Elizabeth O. Joel ${ }^{2}$ \\ ${ }^{1}$ Obafemi Awolowo University, Nigeria \\ ${ }^{2}$ University of Ibadan, Nigeria
}

\begin{abstract}
Consumption awareness of dairy products is increasing with population in Nigeria. Hence, need for bacteriological evaluation of some commonly consumed products. About $10.0 \mathrm{~mL}$ of raw milk (RM) sample was aseptically collected from 50 randomly selected cows during general milking into each sterile bottle. Wara was also sampled from the indigenous producers while yoghurt $(\mathrm{Y})$ samples were sourced from a reputable food company in Ibadan. Aseptic technique was maintained. $1.0 \mathrm{~mL}$ each of serially diluted homogenized bulked RM, Y and macerated wara was pour-plated on plate count agar (PCA) to estimate the total microbial load (TML). The experiment was replicated four times. Viable counts between 30 and 300 colonies per plate were enumerated. Pure bacterial culture was obtained on nutrient agar (NA). Individual isolates were screened morphologically and biochemically for identification. Proximate compositions were also determined. TML was highest (2.8 $\mathrm{x} 106 \mathrm{cfu} / \mathrm{mL})$ in the RM with a significant difference $(\mathrm{p}<0.05)$ across all samples. About 80.0\% of the isolates produced haemolysin, indicating pathogenicity. RM recorded highest moisture (89.83\%) and Ash (0.91\%) while wara had the highest \% values for fat (0.38), protein (12.10) and carbohydrates (10.80) respectively. Different processing methods involved had varying significant suppressing effect on the total number of microbial contaminant.
\end{abstract}

\section{Assessment of the Quality Losses of Fruits and Vegetables during Transportation}

\author{
Ayman H. Amer Eissa ${ }^{1,2, \bullet, \grave{a}}$ and Mostafa M. Azam ${ }^{1,2}$ \\ ${ }^{1}$ King Faisal University, Saudi Arabia \\ ${ }^{2}$ Minoufya University, Egypt
}

\begin{abstract}
The protection of the fruits and vegetables quality in the chains from harvesting to marketing is very important. Vibration often causes some damages to perishable fruits and vegetables in transportation and reduces their quality. Fruits and vegetables injury due to vibration is related to the transportation characteristics of vehicles, packaging boxes and the conditions of the roads. The objective of this study was to investigate the effectiveness of packaging paper by different materials to decrease the damages of vertical vibration on the quality of apple and potato tuber during transportation. The experiments were carried out to measure the dynamic behavior of apple and potato tuber during transporting. The experimental program included the dynamic
\end{abstract}


study online. Operational modal parameter estimation was made based on acquired data for three types of cushioning materials (foam-net, paper-wrap and without (control)). To assess the dynamic behavior of package and the damage of apple and potato tuber due to transient vibration during transporting and the possible effect due to the damage of apple and potato tuber in package. The paper- wrap and foam-net cushioning materials interaction is also studied. Nearly all the examined fruit showed deterioration due to vibro-pressure and friction stresses mainly consisting of translucent or darkened bruises and streaks. The results obtained in range control package shows that increasing damage volume compared to paper-warp and foam-net package, may be due to closing harmonic frequency of mechanical element with natural frequency of apple, potato tuber and this need much attention in design the system for fruit transport trucks in attempt to keep the resonance frequencies of fruit away from exciting frequency of rotating system.

\title{
Directly Filleted Atlantic Salmon - Effect of Rinsing Regime on Color and Stability of Carotenoids in Fillet Surface
}

\author{
Jørgen Lerfall ${ }^{1, \varpi, \varkappa, ~ K a r s t e i n ~ H e i a ~}{ }^{2}$, Bjørn Tore Rotabakk ${ }^{2}$ and Bjørn Roth ${ }^{2}$ \\ ${ }^{1}$ Sør-Trøndelag University College, Norway \\ ${ }^{2}$ Nofima AS, Norway
}

\begin{abstract}
Today's slaughtering and processing method for Atlantic salmon has reached its limit when it comes to using large tanks of RSW (refrigerated sea water) and buffer before and during a stepwise processing regime. To overcome this problem, the salmon industry must, like the poultry industry, move towards automated online production. Automated online production includes rapid processing of the animal, where the power consumption (cooling and transport) can be focused on the meat only and not the surrounding water/ice and carcass. Cutting fillets off, without gill cutting and gutting, will increase needs for effective rinsing of fillets. Rinsing with water to remove blood from fillets is not common practice and may affect the color and carotenoid stability in the fillet surface. One important task is to investigate effects of different rinsing regimes throughout processing. Rinsing time, fillet direction (skin up - skin down) and sweet or salt water rinsing are important factors to optimize to produce a fillet of high quality. The presented study was set up to measure effects of these factors on color and carotenoid stability indirectly filleted Atlantic salmon. The study shows that both fillet color and contents of carotenoids were significantly affected by the different rinsing regimes applied. Hence, the study concluded that optimization of the rinsing process is essential to produce high quality fillets of directly filleted Atlantic salmon.
\end{abstract}

\section{Soluble Gas Stabilization (SGS) Process to Inhibit Microbial Grown of Non-Respiring Food}

\author{
Gabriel Junqueira Cabral ${ }^{1}$, Lenilton Santos Saores ${ }^{1}$, Marieli de Lima ${ }^{1}$, Jonhy T. Teleken ${ }^{1}$, Bruno Augusto M. Carciofi ${ }^{1}$, \\ Bjørn Tore Rotabakk², Morten Silvertvik ${ }^{2}$ and Alcilene Rodrigues Monteiro Fritz ${ }^{1, \bullet, 2}$
}

${ }^{1}$ Federal University of Santa Catarina, Brazil

${ }^{2}$ Nofima AS, Norway

\begin{abstract}
Soluble Gas Stabilization (SGS) is a treatment aiming to dissolve $\mathrm{CO}_{2}$ in food products. As consequence, it can inhibit the bacterial growth and extend the shelf-life of these products. In contact with the food, the $\mathrm{CO}_{2}$ solubilizes in both aqueous and fat phases, reducing the $\mathrm{pH}$ and acting as an antibacterial. The goal of this study was to enhance the shelf-life of non-respiring foods like oysters, mussels and minas fresh cheese, by Appling SGS process carried out under different pressure and temperature. $\mathrm{CO}_{2}$ concentration into the food was determined by manometric method and the food shelf-life was evaluated by the microbial growth and the physicochemical properties, such firmness, exudation, and pH. After the SGS treatment (absorption step), the $\mathrm{CO}_{2}$ desorbed quickly into the packaging, however this time to reach the maximum concentration in the packaging headspace change according the food. Samples treated with SGS has been increased their shelf-life, mainly by reducing the bacterial growth rate, for the three studied food products, when comparing with control samples packaged under atmospheric air, mainly by reducing the bacterial growth rate, for the three products studied. The majority of physicochemical was not significantly affected. The SGS process showed to be a useful tool to improve the shelf-life of different protein non-respiring food products.
\end{abstract}




\title{
Preservation of Highbush Blueberry Fruit (Vaccinum corymbosum L.) using Novel Ultrasound Assisted Drying Techniques
}

\author{
Karolina Siucińska ${ }^{1, \bullet,\llcorner, ~ D o r o t a ~ K o n o p a c k a ~}{ }^{1}$ and Ryszard Parosa ${ }^{2}$ \\ ${ }^{1}$ Research Institute of Horticulture, Poland \\ ${ }^{2}$ Promis-Tech, Poland
}

\begin{abstract}
The role of high bush blueberry fruit in the world wide commercial production of horticulture commodities is continually increasing, as they are not only attractive and palatable, but additionally the fruit has been proven to offer several nutritional benefits, mainly due to their high antioxidant activity. Having a limited growing season, the fruit has to be preserved. One of the most popular preservation methods is drying, preferably after a previous taste profile adjustment by osmotic pretreatment. Although several drying methods for blueberry drying are available, sustainable and effective methods for blueberry preservation would be advisable. Using the novel hybrid drier, where classic convection drying with a forced movement of dried material is supported by microwave energy and an intensive stream of ultrasound, the procedure for the drying of blueberries, both previously osmo-treated or only frozen, was developed. The abovementioned drying technique was based on the sequential use of ultrasound, microwaves and hot air energy, with alternating cycles of high $\left(60{ }^{\circ} \mathrm{C}\right)$ and low $\left(20{ }^{\circ} \mathrm{C}\right)$ temperature, assisted by air-borne ultrasound $(200 \mathrm{~W}, 25 \mathrm{kHz})$ with microwave energy applied at the final stage of drying. It was confirmed that hybrid drying can be used for osmotically dehydrated fruits, which additionally reduced drying time by $50 \%$ when compared with convective drying (from $6 \mathrm{~h}$ to $3 \mathrm{~h}$ ). Moreover, our method resulted in an attractive ready-to eat dried product highly appreciated by the panelists in sensory assessment that can also be applied to sour cherries.
\end{abstract}

Acknowledgement: This work had been prepared within the 'BIOSUSZ' PBS Project financed by the National Centre for Research and Development (PBS1/A8/13/2012).

\section{Comprehensive Extraction of Oil and Lignocellulose from Soy Sauce Residue}

\author{
Pengfei Chen ${ }^{\varpi, \iota, ~ H a n g ~ S o n g, ~ X u e t i n g ~ F e n g, ~ H u i m i n ~ Z a n g, ~ S h u n ~ Y a o ~ a n d ~ Y i n g y i n g ~ Y a n g ~}$ \\ Sichuan University, China
}

\begin{abstract}
Soy sauce residue (SSR), as an industrial by-product of soy sauce manufacturing process, is classified as waste biomass mainly containing oil and lignocellulose. In order to reduce the burden of the waste treatment of the soy sauce industry and/or bring some benefits to soy sauce producers, the extraction of oil and lignocellulose in SSR was investigated. Firstly, orthogonal experiments were used for optimizing the extraction technology of oil from SSR. Moreover, the composition and relative content of fatty acids in oil was analyzed by GC/MS. Results indicated that the optimal yield of oil reached $80.8 \%$ and the relative content of palmitic acid, linoleic acid, oleic acid, palmitic acid in the oil was $46.90 \%, 33.26 \%, 14.87 \%, 4.90 \%$, respectively. Then, the contents of cellulose, hemicellulose and lignin in deoiled SSR were determined. On the basis, lignocellulose was extracted from the deoiled SSR. To explore an extract method that can be potentially used in industrial application, 1-butyl-3methylimidazolium hydroxide (BMIMOH), a kind of typical basic alkaline IL, was employed to substitute for sodium hydroxide and the reusability of the IL was also studied. This study presents a promising approach and new process to make use of soy sauce residue and other similar waste biomass.
\end{abstract}

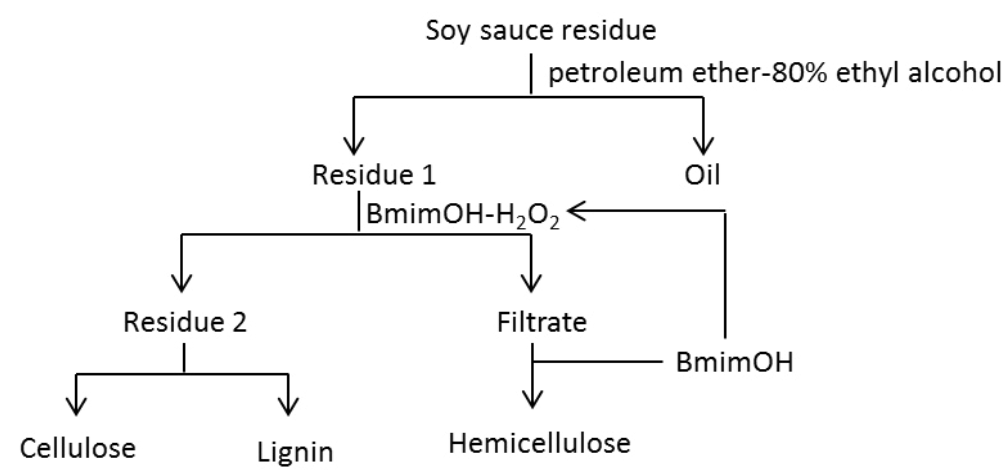




\title{
Varietal Thiols in Wine: A Review on Their Aromatic Contribution and Their Liberation Mechanism from the Precursors Present in Grapes and Musts
}

\author{
Aurélie Roland ${ }^{1}$, Hugo Bonnaffoux ${ }^{2,3}$, Florine Cavelier ${ }^{2}$ and Rémi Schneider ${ }^{1,3, \square, 2}$ \\ ${ }^{1}$ Nyseos, France \\ ${ }^{2} I B M M$, Université Montpellier, France \\ ${ }^{3} I F V-U M T$ Qualinnov, France
}

\begin{abstract}
Among light and heavy sulphur compounds, three varietal thiols (4-mercapto-4-methylpentan-2-one (4MMP), 3-mercaptohexan-1-ol (3MH) and 3-mercaptohexylacetate (A3MH) have been identified in the late 90's as key odorant compounds of wine (Sauvignon Blanc). These thiols are released during the alcoholic fermentation (AF) from precursors initially present in grapes or musts. Different biogenetic pathways are reported for the $4 \mathrm{MMP}$ and $3 \mathrm{MH}$ formation, whereas the A3MH came from the $3 \mathrm{MH}$ acetylation by yeast. The first two pathways involved cysteninylated and glutathionylated $\mathrm{S}$-conjugates that are cleaved by the yeast $\beta$-lyase activity during AF. A third pathway has been also identified from (E)-2hexenal but also (E)-2-hexenol, although the sulphur donor hasn't been yet identified. The calculation of 3MH levels originating from those pathways could explain from 10 to $65 \%$ of the $3 \mathrm{MH}$ present in wine. Thus a large field of investigation is opened to better understand the thiols biogenesis. Recently, some new derivatives (aldehydic forms, $\mathrm{SO}_{2}$-combined forms, glutathione derivatives) have been identified. Some viti cultural techniques favored thiols precursors accumulation in grapes or musts, such as the vine water status and the nitrogen fertilization management (soil fertilizer or foliar spraying), or oxidation mechanisms during the must elaboration. However, fermentation conditions could hugely influence thiols release: yeast choice, temperature and yeast nitrogen nutrition. If lots of studies focused on the yeast thiol release capacity s, let's keep in mind that the conversion yields reported never exceed 10\%. Surprisingly, few studies were dedicated to the thiols precursors transport in the yeast. The General Amino acid Permease 1 (GAP1) is known to be the main transport system for cysteinylated precursors. Its regulation by the ammonium levels in must explained that yeast nutrient composition $\left(\mathrm{NH}_{4}^{+} /\right.$amino acids ration) and time of addition are key parameters. For glutathionylated precursors transport in the cell and their release, mechanism only hypothesis can be formulated
\end{abstract}

\section{Effect of Drying and Frying Conditions on Physical and Chemical Characteristics of Fish Maw from Swim Bladder of Seabass (Lates calcarifer)}

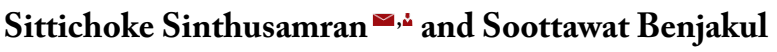 \\ Prince of Songkla University, Thailand
}

\begin{abstract}
Swim bladder is generated as by-product during evisceration. It has been used for fish maw production, in which several processing parameters determine the characteristics or quality of resulting fish maw. The present study aimed to investigate the characteristics of fish maws from seabass swim bladder as influenced by drying and frying conditions. Expansion ratio and oil uptake content of fish maw increased as the moisture content of swim bladder increased $(\mathrm{P}<0.05)$. Nevertheless, the expansion ratio of fish maw became decreased as moisture content was higher than $15 \%$. $\mathrm{L}^{*}$-value decreased, whilst $\mathrm{a}^{*}$ and $\mathrm{b}^{*}$-values of fish maw increased with increasing moisture content. When pre-frying and frying temperatures increased, the expansion ratio of fish maw increased $(\mathrm{P}<0.05)$. However, expansion ratio decreased when the frying was performed at temperature higher than $200{ }^{\circ} \mathrm{C}$. The oil uptake content of fish maw with frying temperatures of 180 and $200{ }^{\circ} \mathrm{C}$ was in the range of $45.11-57.81 \%$, whereas the lower contents (37.8.1-41.72\%) were found in those having frying temperatures of $220-240{ }^{\circ} \mathrm{C}$. The texture of fish maw became harder, and fracturability decreased with increasing frying temperature when frying was carried out at $200{ }^{\circ} \mathrm{C}$. Drying temperatures, moisture content, pre-frying and frying temperatures were the factors influencing the characteristics and properties of fish maws from seabass swim bladder. Fish maw could be prepared by pre-frying swim bladder, dried at $60{ }^{\circ} \mathrm{C}$ to obtain $15 \%$ moisture content, at $110{ }^{\circ} \mathrm{C}$ for $5 \mathrm{~min}$, followed by frying at $200{ }^{\circ} \mathrm{C}$ for $20 \mathrm{sec}$. Fish maw is the puffed swim bladder, an internal gas-filled organ found in most fish. It has long been consumed as a popular soup in China and South-East Asia. The important factors affecting the quality of fish maw are drying and frying processes. The objective was to investigate the effect of moisture content of swim bladder and frying conditions on characteristics and properties of fish maw.
\end{abstract}




\title{
Efficient Image Analysis Method to Evaluate Ripening Process of Fruits and Correlating with Physicochemical and Microscopy Techniques
}

\author{
Stefany Cárdenas-Pérez ${ }^{1, \varpi, ¿}$, Jorge Chanona-Pérez ${ }^{1}, J_{u a n}$ Méndez-Méndez $^{2}$ and Georgina Calderón-Domínguez ${ }^{1}$ \\ ${ }^{1}$ Escuela Nacional de Ciencias Biológicas Instituto Politécnico Nacional,México \\ ${ }^{2}$ Centro de Nanociencias y Micro y Nanotecnologías- Instituto Politécnico Nacional, México
}

\begin{abstract}
At this time the agro industry requires non-destructive and economic methods which can allow a reliable assessment of the ripeness degree in climacteric fruits, since ripeness is an important quality attribute that has traditionally been evaluated by visual human inspection and destructive physicochemical methods. Recently, non-destructive technologies have emerged in order to have a rapid and efficient evaluation at low cost. Therefore, the aim of this work is to evaluate the degree of ripening and quality of apple (Golden delicious) by means of a computer vision system (CVS) based on the color analysis correlating with colorimeter data as well as physicochemical and microscopy studies with Environmental Scanning Electron Microscopy (ESEM) and Atomic Force Microscopy (AFM), techniques which were used to follow the changes that occur in the cell wall of apple tissue during its ripening. A set of 56 apples was used to evaluate the ripening process for CVS, physicochemical and microscopy analysis. The physicochemical analysis helped to describe the process of apple ripening, especially the kinetic of maturity. The duration of apple ripening is already reported to be 32 days after the harvest (Cen et al. 2013). The color parameters $\left(\mathrm{L}^{*}, \mathrm{a}^{*}\right.$ and $\left.\mathrm{b}^{*}\right)$ extracted from CVS were efficient to describe the changes in apple skin color during the storage time. The results obtained from CVS shown a good correlation of the data obtained by colorimeter, physicochemical, ESEM and AFM. These studies were useful to describe the maturation kinetic of apple tissue.
\end{abstract}

\section{Impact of Starter Culture on the Aroma Profile and Sensory Attributes of Barley Malt Beverages Produced by Lactic Acid Fermentation}

\author{
Sorelle Nsogning Dongmo ${ }^{a, 2}$, Bertram Sacher, Hubert Kollmannsberger and Thomas Becker \\ Technische Universität München, Germany
}

\begin{abstract}
Due to society quest for health promoting foods, fermented malt based beverages are gaining interest as alternative to dairy products, and non-alcoholic beverages. Their sensory profile is decisive for the future market, since flavor improvement was claimed by consumers. In order to improve the aroma profile, initial approach is the elucidation of the aroma composition of these beverages, and the changes during fermentation. We described differences in aroma profile of barley malt beverages produced by fermentation with six selected starter cultures of lactic acid bacteria. After aroma compounds isolation from fermented beverages by three different techniques, identification was performed for quantitation by means of gas chromatography- mass spectrometry/olfactometry. Internal standard calibrations, odor activity value calculations, aroma extract dilution analyses, and sensory characterization were considered. Diverse aroma compounds identified were found to be formed during fermentation although, some derived from barley malt wort. However, only few of them, present at concentrations higher than the odor detection thresholds were determinant for the aroma profile of the beverages. Variation in starter culture led to beverages with different sensory attributes. Similarly, key odorants with fruity pleasant odor quality were found to be specific to the starter culture. Our findings revealed potential species of lactic acid bacteria as starter cultures which tend to produce beverages with fruity and pleasant flavor. This indicates that suitable choice of lactic acid bacteria starter culture could improve the aroma profile of cereal beverages.
\end{abstract}

\section{Physicochemical Parameters and Anthocyanins Kinetic Degradation Modelling from Blood Orange Juice as Affected by Ascorbic Acid Fortification During Pasteurisation}

\author{
HocineRemini ${ }^{1}$, Sabiha Achat ${ }^{1, \bullet, \varkappa}$, Hayate Haddadi-Guemghar ${ }^{1}$, Lila Boulekbache-Makhlouf ${ }^{1}$, Sonia Oukhmanou- \\ Bensidhoum $^{1}$, Nadia Bouaoudia-Madi ${ }^{1}$, Lamia Haddache ${ }^{1}$, Sofiane Dairi ${ }^{1}$, Farid Dahmoune ${ }^{1,2}$ and KhodirMadani $^{1}$
}

${ }^{1}$ Université de Bejaia, Algeria

${ }^{2}$ Université de Bouira, Algeria 


\title{
Abstract
}

The "pure fruit juice" or " $100 \%$ pure juice" are beforehand the most "natural". They are obtained by a simple squeezing (within 24 hours after harvesting) of blood orange carefully selected; without any addition: no coloring, no preservatives added (natural or synthetic), no water or sugar added (blood orange pressed and nothing else), for which only a fortification by vitamin $\mathrm{C}$ and a pasteurization is permitted. For that, the variation of anthocyanin and physico-chemical parameters $(\mathrm{pH}$, titratable acidity, and soluble solid content) of the blood orange juice (Citrus sinensis [L.] Osbeck) during pasteurization was monitored at 60 to $90{ }^{\circ} \mathrm{C}$. The effect of ascorbic acid fortification (at $100 \mathrm{ppm}$ ) and temperature/time pasteurization on the kinetic behavior of anthocyanin degradation was determined. The total anthocyanin content was assessed by the $\mathrm{pH}$ differential method. Firstorder reactions models best fit the anthocyanin kinetic degradation and three models (Arrhenius, Eyring and Ball) were used to assess the temperature-dependent degradation. Ascorbic acid fortification is the most influential factor on anthocyanin kinetic degradation, while no significant changes on the physico-chemical parameters were revealed during pasteurization at all temperatures.

Acknowledgements: The authors gratefully acknowledge the Algerian Ministry of Higher Education and Scientific Research for funding the study.

\section{Effect of Ultra - Violet (UV) Irradiation on the Postharvest Control of Cowpea Weevil and the Proximate Composition of Cowpea Seeds}

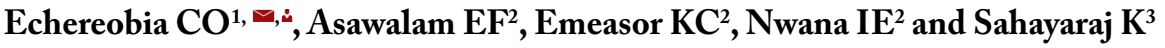 \\ ${ }^{1}$ Federal University of Technology Owerri, Nigeria, \\ ${ }^{2}$ Michael Okpara University of Agriculture, Nigeria \\ ${ }^{3}$ St Xavier's College (Autonomous), Tamil Nadu, India
}

\begin{abstract}
Cowpea is a cheap source of protein in Nigeria. However, insect pests like Cowpea weevils cause quantitative and qualitative losses to cowpea seeds thereby reducing the nutritive values. Consequently, experiments were conducted at the Crop Protection Research Centre, St Xavier's College (Autonomous), Palayamkottai, India under laboratory conditions $\left(28 \pm 2{ }^{\circ} \mathrm{C}, 70-75 \%\right.$ RH and 11:13h photoperiod) to determine the effect of UV irradiation on the postharvest control of Cowpea Weevil and the proximate composition of cowpea seeds. Five pairs of 2 day old cowpea weevil collected from stock culture were kept in a Petridish containing 50 wholesome seeds (Ife brown variety) and exposed to UV rays for 0, 5, 10,15 and 20 mins. The experiment was conducted using Completely Randomized Design (CRD) replicated five times. Results showed that UV irradiation was effective in protecting cowpea seeds against cowpea weevils and did not significantly affect the protein and carbohydrate contents of cowpea.
\end{abstract}

\section{Poster Abstracts}

\section{Sorption Isotherms of Microcapsules of Betalains Using Modified QPM Starch as Wall Materials by Spray Drying}

\author{
Amira Daniela Calvo-López ${ }^{1, \varpi, \imath, ~ F e r n a n d o ~ M a r t i ́ n e z-B u s t o s ~}{ }^{1}$ and Pedro Alberto Vázquez-Landaverde ${ }^{2}$ \\ ${ }^{1}$ Centro de Investigación y de Estudios Avanzados del, Instituto Politécnico Nacional (CINVESTAV-Unidad Querétaro), México \\ ${ }^{2}$ Centro de Investigación en Ciencia Aplicada y Tecnología Avanzada (CICATA), Instituto Politécnico Nacional (IPN), México
}

\begin{abstract}
The microencapsulation principle is the creation of a barrier between the components inside the particle and the environment. Microencapsulation by spray drying is the process most commonly used in the food industry. Particles production involves the formation of an emulsion using carrier materials and the active material (natural dyes), followed by atomization of the emulsion in a drying chamber with circulation of hot air (Murúa, 2009). The aim of this work was the microencapsulation of dye extracted from the fruit "jiotilla" (Escontria chiotilla [Weber] Britton \& Rose) using maltodextrins and modified QPM starch as wall materials by spray drying. The pigments of "jiotilla" are derived from the betalains, natural water-soluble pigments composed of two structural groups: betacyanins (reddish-purple) and betaxanthins (orange-yellow). Sorption isotherm at 25, 30 and 35 ${ }^{\circ} \mathrm{C}$ (gravimetric method) was measured at differing water activities to evaluate the stability of the encapsulated betalains. Six different assays were performed: succynilated starch modified by extrusion (SUCC) in blends with maltodextrins (M): a) SUCC
\end{abstract}


0\% M, b) SUCC 10\% M, c) SUCC 20\% M, d) SUCC 30\% M, e) N-LOK (as control), and f) partially hydrolyzed starch by extrusion (HIDRO). The drying conditions were $170{ }^{\circ} \mathrm{C}$ as inlet temperature and $90{ }^{\circ} \mathrm{C}$ outlet temperature with a feed rate of $10 \mathrm{~mL} / \mathrm{min}$ and solids content of $10 \%$. Sorption isotherms determined that the HIDRO starch provides better protection to the dye due to increases the water activity to a lesser extent than other assays. These microcapsules can be used to providing color to foodstuffs with high water activities, N-LOK starch was solubilized to high water activities $(>0.8)$ causing the total destruction of the microcapsules, the succynilated starch has a damping effect of moisture to high water activities. When increasing the content of maltodextrins in the wall material, the microcapsules were more susceptible to degradation by moisture.

\title{
Acetylated Starches as Shell Materials for Encapsulation of Folic Acid and Ferrous Sulfate by Spray Drying and its Functionality in Nixtamalized Maize Flours and Tortillas
}

\author{
Fernando Martínez Bustos ${ }^{\varpi, \AA}$ and Alejandro Cruz Sánchez \\ Centro de Investigación y de Estudios Avanzados del Instituto Politécnico Nacional (CINVESTAV-Unidad Querétaro), México
}

\begin{abstract}
The deficiency of folate and iron are public health problems in developing countries, causing anemia, heart disease and digestive and intestinal disorders. Some foods are fortified in some developing countries. Folate and iron fortification of food is generally considered to be the best long-term strategy to increase its absorption. However, there are numerous challenges that the health national programes face implementing effective food fortification: iron in its most absorbable forms, is highly reactive that may adversely affect the sensory properties of fortified foods and precipitated as insoluble iron complexes. Also, folic acid is very sensitive to temperature changes during storage in fortified foods. The aim of this investigation was to to develop microcapsules of folic acid and ferrous fumarate using acetylated normal and waxy maize starches as wall materials and to evaluate the functional and sensorial properties of fortified nixtamalized maize flour and tortillas. Viscosity and color of traditional nixtamalized maize flours were not affected with the addition of microcapsules with folic acid or ferrous fumarate. The values of adhesivity, tensile strength and moisture content of fortified masa were similar to these values of masa prepared by the traditional method (control). Functional characteristics (rollability, puffing, tensile strength, cutting force and moisture content) and encapsulated folic acid and ferrous fumarate contents of fortified tortillas were not modified during storage (room temperature and refrigerated). Tortillas produced with this enrichment strategy displayed similar functional and sensorial properties than traditional tortillas.
\end{abstract}

\section{Extraction of Betalains from Pitaya (Stenocereuspruinosus) Fruit and Their Encapsulation by Spray Drying Using Modified Potato Starch}

\author{
Lilia Vargas-Campos ${ }^{1, \bullet}$, ¿, Salvador Valle-Guadarrama ${ }^{1}$, Fernando Martínez-Bustos², Amira D. Calvo-López², Yolanda \\ Salinas-Moreno ${ }^{3}$ and Consuelo Lobato-Calleros ${ }^{1}$
}

${ }^{1}$ Universidad Autónoma Chapingo, México

${ }^{2}$ Centro de Investigación y de Estudios Avanzados del Instituto Politécnico Nacional (CINVESTAV-Unidad Querétaro), México

${ }^{3}$ Instituto Nacional de Investigaciones Forestales, Agricolas y Pecuarias

\begin{abstract}
Betalains are natural red colorants, appreciated by food industry. However, these substances are sensible to environmental factors such as light, oxygen, temperature, and water activity. Due to this a protection strategy should be applied.The objective of this research was to extract, purify, and encapsulate betalains obtained from pitaya (Stenocereus pruinosus) fruit in order to favor their use as colorant of food products. A first phase was conducted where betalains were extracted using an ethanol-water solution, and were purified by the aqueous two phase extraction method to remove sugars which can cause fermentation or caramelization. The sugars separation efficiency was $81 \%$. A second phase was conducted to encapsulate betalains. Natural potato starch was modified through hydroxylation and also by esterification with sodium tripolyphosphate (phosphated) and n-octenylsuccinican hydride (succinated) by thermoplastic extrusion to favor its use as encapsulating agent. The microencapsulation efficiency was calculated in percentage as the ratio of the difference between total betalains and superficial betalains to total betalains. Besides, the microencapsulation yield was determined as the ratio of encapsulated mass to the mass of solids in the emulsion. The values of efficiency and yield of microencapsulation with succinated starch were $48.72 \%$ and $48.60 \%$, respectively, while values obtained with phosphated starch were $81.90 \%$ and $49.30 \%$. These contrasted with the quantities of $86.52 \%$ and $63.30 \%$, respectively, obtained when a commercial modified starch was used as control. It was concluded that phosphated starch offers potential to be
\end{abstract}




\title{
Determination of Heavy Metals Concentration in Commonly Consumed Traditional Herbs in the United Arab Emirates
}

\author{
Rania Dghaim ${ }^{\varpi, ~ «, ~ S a f a ~ A l ~ K h a t i b ~ a n d ~ M u n a w w a r ~ A l i ~ K h a n ~}$ \\ Zayed University, Dubai, United Arab Emirates
}

\begin{abstract}
Herbs are extensively consumed in the (UAE) for their flavoring and medicinal properties. Contamination of herbs with heavy metals has been reported in several studies. This study aimed at determining the concentration of heavy metals in selected traditional herbs commonly consumed in the (UAE). A total of 81 samples of seven herbs: parsley (Petroselinum crispum), basil (Ocimum basilicum), sage (Salvia officinalis), oregano (Origanum vulgare), mint (Mentha spicata), thyme (Thymus vulgaris), and chamomile (Matricaria chamomilla) were purchased from the local market in Dubai, and analyzed for their cadmium, lead, copper, iron and zinc contents. Microwave-assisted digestion was applied for the dissolution of the samples. Heavy metals concentration was determined in the herb samples using Atomic Absorption Spectrometry (AAS). Metals were found to present in varied concentrations in the herb samples. The concentration ranges were found (less than $0.1-1.11 \mathrm{mg} \cdot \mathrm{kg}^{-1}$ ) for cadmium, (less than 1.0 - $\left.23.52 \mathrm{mg} \cdot \mathrm{kg}^{-1}\right)$ for lead, $\left(1.44-156.24 \mathrm{mg} \cdot \mathrm{kg}^{-1}\right)$ for copper, $\left(12.65-146.67 \mathrm{mg} \cdot \mathrm{kg}^{-1}\right)$ for $\mathrm{zinc}$, and $\left(81.25-1101.22 \mathrm{mg} \cdot \mathrm{kg}^{-1}\right)$ for iron. The findings of the study suggest that most of the analyzed herbs contained unsafe levels of heavy metals that exceeded the World Health Organization (WHO) permissible limits (PL). Contamination of herbs with heavy metals beyond internationally accepted limits poses a potential risk on consumer's health.
\end{abstract}

\section{Microbiological Evaluation of a Small Scale Processing for Sugarcane Juice}

\author{
Rodrigo Petrus $^{\varpi, ょ, ~ C a r i n e ~ S i l v a, ~ M a r t a ~ K u s h i d a, ~ F a b i o ~ G a l l o, ~ L a u r a ~ B o m d e s p a c h o, ~ M a r i a ~ L u i z a ~ S i l v a ~ a n d ~ M a ́ r c i a ~ V e r w e i j ~}$ \\ University of São Paulo, Brazil
}

\begin{abstract}
Investigation was carried out to evaluate control points in a pilot plant designed for sugarcane juice processing. The juice was extracted in a stainless steel electric cylinder mill and then acidified with citric acid until attaining the $\mathrm{pH}$ of 4.3 . Next it was pasteurized in a plate heat exchanger at $95^{\circ} \mathrm{C} / 30 \mathrm{~s}$ and then cooled to $10{ }^{\circ} \mathrm{C}$ before being filled into plastic bottles and induction sealed. Product's filling was performed in an ISO class 5 unidirectional air-flow cabin. Bottles were decontaminated by $0.05 \%$ peracetic acid (PAA) spray, at $45^{\circ} \mathrm{C}$ for 20 s. Seals and caps were sterilized at $121^{\circ} \mathrm{C} / 15 \mathrm{~min}$. Three batches of acidified sugarcane juice were carried out. The qualities of the raw material ( $\mathrm{pH} 5.1$ and $22.3^{\circ} \mathrm{Brix}$ ), of the rinse water of the processing and filling line (after sanitation with $0.1 \% \mathrm{PAA}$ at $50{ }^{\circ} \mathrm{C}$ for $40 \mathrm{~min}$ ), of the packaging and of the end product, were all microbiologically evaluated. The total aerobic mesophilic and molds and yeasts mean counts in natural fresh sugarcane juice were $6.3 \mathrm{log} \mathrm{CFU} / \mathrm{mL}$ and $5.2 \log \mathrm{CFU} / \mathrm{mL}$, respectively. The mesophilic and molds and yeasts mean counts taken, in both rinse water samples of the processing line and the bottles, were lower than $1 \log \mathrm{CFU} / \mathrm{mL}$. The mesophilic and molds and yeasts mean counts in acidified and pasteurized sugarcane juice were 2.6 and lower than $1 \log \mathrm{CFU} / \mathrm{mL}$, respectively. The findings indicated that the procedures that were evaluated met standards for acidified sugarcane juice to be produced then stored under refrigeration.
\end{abstract}

\section{Shelf-life of Standardized and Pasteurized Sugarcane Juice}

\author{
Rodrigo Petrus $^{\varpi, \varkappa}$, Ivana Andrade and Fabio Gallo \\ University of São Paulo, Brazil
}

\begin{abstract}
The shelf-life (SL) of fresh sugarcane juice is quite limited because of the high rates of microbiological and enzymatic reactions which take place after juice's extraction. This study was undertaken to estimate the SL of standardized and pasteurized sugarcane juice, aseptically packaged in polyethylene terephthalate (PET) bottle and stored under ideal, commercial and abusive temperature conditions. The $\mathrm{pH}$ of juice was reduced to approximately 4.0 by addition of citric acid, and soluble solids adjusted near $19.5^{\circ}$ Brix. Three batches of standardized sugarcane juice were pasteurized at $95{ }^{\circ} \mathrm{C} / 30 \mathrm{~s}$ and cooled at $10{ }^{\circ} \mathrm{C}$. Each processed batch was divided into four parts, stored at $4,8,12$ and $-18^{\circ} \mathrm{C}$ (control) in the dark. Physicochemical, microbiological
\end{abstract}


and sensory tests were carried out to estimate the SL of the product in each storage temperature. Bacteria and fungi psychotropic counts equivalent to 104 and $103 \mathrm{CFU} / \mathrm{mL}$, respectively, were established as maximum acceptable levels. Nine-point hedonic scale tests were applied to a team of fifty panelists who evaluated the appearance and the flavor of juice. Averages of above five points and percentages of acceptance of above $60 \%$ were adopted as limit values for estimating the sensory stability. The average SL of juice stored at 4,8 and $12{ }^{\circ} \mathrm{C}$ were 74,71 and 21 days, respectively. The sensory quality decrease limited the product's SL. The temperatures of 4 and $8^{\circ} \mathrm{C}$ proved to be effective in maintaining an acceptable level of quality for a period compatible with the consumer market demand.

\title{
Development of an Isotonic Drink with Ricotta Cheese Whey
}

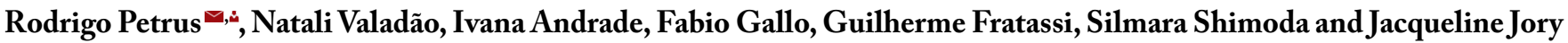 \\ University of São Paulo, Brazil
}

\begin{abstract}
Ricotta cheese whey $(\mathrm{RCW})$ is a by-product of the dairy industry and it is usually discarded or used as animal feed. This study focused on RCW to develop an isotonic drink. Lactase was added to RCW then kept at $7{ }^{\circ} \mathrm{C}$ for 24 hrs. The pre-treated $\mathrm{RCW}$ was pasteurized at $75^{\circ} \mathrm{C} / 15 \mathrm{~s}$, filled into high density polyethylene bottles, stored at $0{ }^{\circ} \mathrm{C}$ and then used to develop the isotonic formulation. In order to choose the best flavour, Rank Preference tests with seven commercial samples with different flavours were performed. Dozens of experimental formulations were tested; the amount of ingredients and additives (RCW, deionized water, sucrose, sodium chloride, citric acid, colorant and flavouring) was varied based on the preference of sixty panelists. Just Right Scale tests were performed to evaluate color, odor, sweet, salty and sour tastes. Nine-point Hedonic Scale tests were simultaneously performed. Measurements of $\mathrm{pH}$, soluble solids content, osmolality, and sodium and potassium levels were also performed. The tangerine flavor was preferred by the panelists $(p \leq 0.05)$ and then selected to develop the isotonic formulation. After conducting ten sessions of sensory analysis and physicochemical tests during approximately six months, an isotonic formulation accepted by the sensory panel (mean score of 6.3) was achieved. The results of physicochemical tests were in compliance with Brazilian Food Regulation, with pH 3.1, $6.3{ }^{\circ} \mathrm{Brix}, 306 \mathrm{mOsm} / \mathrm{L}$, and 500 and $650 \mathrm{mg} / \mathrm{L}$ sodium and potassium, respectively. The findings indicate that RCW could be a technologically viable alternative to produce an isotonic drink.
\end{abstract}

\section{Selective Identification and Quantification of Saccharin by Liquid Chromatography and Fluorescence Detection}

\author{
Sergio N.F. Bruno ${ }^{1, \varpi, \imath,}$, Carlos R. Cardoso ${ }^{1}$, Márcia Mosca A. Maciel ${ }^{1}$, Lidmila Vokac ${ }^{1,2}$ and Ademário I. da Silva Junior ${ }^{2}$ \\ ${ }^{1}$ Advanced Laboratory Service - SLAV-RJ/LANAGRO-MG/MAPA, Brazil \\ ${ }^{2}$ Instituto Federal do Rio de Janeiro (IFRJ), Campus Rio de Janeiro, Brazil
}

\begin{abstract}
High-pressure liquid chromatography with ultra-violet detection (HPLC-UV) is one of the most commonly used methods to identify and quantify saccharin in non-alcoholic beverages. However, due to the wide variety of interfering UV spectra in saccharin-containing beverage matrices, the same method cannot be used to measure this analyte accurately. We have developed a new, highly effective method to identify and quantify saccharin using HPLC with fluorescence detection (HPLC-FLD). The excitation wavelength $(250 \mathrm{~nm})$ and emission wavelength $(440 \mathrm{~nm})$ chosen increased selectivity for all matrices and ensured few changes were required in the mobile phase or other parameters. The presence of saccharin in non-diet beverages - a fraud commonly used to replace more expensive sucrose - was confirmed by comparing coincident peaks as well as the emission spectra of standards and samples. This new was efficient, selective, fast and precise, and showed an appropriate sensitivity to the target analyte. Proficiency and recovery tests indicated the accuracy of the method. Its application in an inter-laboratory trial was also successful.
\end{abstract}

\section{Solubility of $\mathrm{CO}_{2}$ in Lamb Meat and Minas Fresh Cheese by Manometric Method}

Fernanda Fidler, Gabriel Junqueira Cabral, Lenilton Santos Saores, Jonhy T. Teleken, Bruno Augusto M. Carciofi and Alcilene Rodrigues Monteiro ${ }^{a, 4}$

Federal University of Santa Catarina, Brazil 


\title{
Abstract
}

Carbon dioxide is a gas with antimicrobial properties and has been used in the modified atmosphere packaging (MAP). Therefore, the $\mathrm{CO}_{2}$ should be applied to lamb meat and cheese due the gas will be absorbed by fat and muscle of meat until the equilibrium. The aim this studied was to determine the solubility of the $\mathrm{CO}_{2}$ in the lamb meat and minas fresh cheese by manometric method. Solubility of $\mathrm{CO}_{2}$ in fresh cheese were determined at 4,7 and $10{ }^{\circ} \mathrm{C}$ and the lamb meat was determinate at 2,6 and $10^{\circ} \mathrm{C}$ at constant volume. For cheese highest solubility value was obtained at $4{ }^{\circ} \mathrm{C}$ which was close to the value observed at $7{ }^{\circ} \mathrm{C}$. This behavior may be explained by the combined effects of temperature and complex phase transitions in fat content, shown by differential scanning calorimetry (DSC) curves. For the lam meat samples highest solubility value was obtained at $2{ }^{\circ} \mathrm{C}$. Both foods studied observed decreased of solubility with increased of temperature. $\mathrm{CO}_{2}$ solubility in fat is influenced by the saturation degree of fatty acids. Studies demonstrate that $\mathrm{CO}_{2}$ solubility in fatty tissues may increase with temperature due to increasing the melted fraction However, it is known that an increase in temperature promotes a decrease in gas solubility due to the greater thermal motion in molecules. $\mathrm{CO}_{2}$ solubility behavior in the Minas Frescal cheese and lamb meat studied in this research could be attributed to the superposition of both effects.

\section{Proteomic Approach of Phage Infection in E. coli O157:H7}

\author{
Maryoris E. Soto Lopez, Regina Célia Santos Mendonça ${ }^{\varpi, \AA, ~ E d v a l d o ~ B a r r o s, ~ L a i ́ s ~ S i l v a ~ B a t a l h a ~ a n d ~ L u i z ~ A u g u s t o ~ A l b i n o ~}$ \\ Viçosa Federal University, Brazil
}

\begin{abstract}
E. coli $\mathrm{O} 157: \mathrm{H} 7$ is the food borne pathogen of highly importance in the medical and food industry. This bacteria belongs to the family Enterobacteracea, which cause approximately 9,000 infections and 600 deaths per year. Bacteriophages have been used as an alternative to antimicrobial resistant $E$. coli $\mathrm{O} 157: \mathrm{H} 7$ with promising results. Bacteriophages are viruses that infect bacteria. They can take over the bacterial machinery and use replicate their genome. After infection, the bacteriophages utilize the host transcriptional apparatus following of DNA injection. At this point, all information contained in the genome of bacteriophage begins to be transcribed and translated by cellular machinery. So, the entire protein expression of the host bacteria focuses only to produce new viral particles. The essential of biocontrol is use a lytic phage to reduce pathogens. Thus, it is necessary understand the protein expression modulation and all proteins involved on the cell death, during infection. For this kind of study, proteomic analyses are necessary. Proteomics are the study of proteomes, which express several proteins of the organism. In this study was observed the protein expression in E. coli O157:H7 modulated by bacteriophage AREG after 15 mins of infection, using as reference $E$. coli $\mathrm{O} 157: \mathrm{H} 7$ in normal conditions. Although the SDS-PAGE electrophorese was used to verify the quality of proteins and how the expression change in the microorganism after the phage infection. Results showed the difference in protein expression of E. coli O157:H7 when it was infected by bacteriophage. In the SDS-PAGE electrophorese gel can be observed that some bands disappear. This can be an indication that some proteins were express in lower quantity than others. Furthermore, novel proteins have been expressed after contact with the bacteriophage.
\end{abstract}

\section{Comparison Between Two Extraction Methods for Obtaining Olive Leaf Extract Rich in Oleuropein}

\author{
Cintia B. Gonçalves ${ }^{\natural, 2}$, Carolina F. S. Cebin Coppa, Roice E. Rosim, Carlos A. F. Oliveira and Christianne E. C. Rodrigues \\ University of São Paulo - FZEA/USP, Brazil
}

\begin{abstract}
Olive leaves are rich in oleuropein, which has been widely studied due to its pharmacological functions. Some methods for obtaining this component have been reported, but the extraction with ethanol has emerged because of its non-toxic characteristics. This study aimed to evaluate the oleuropein yield in extracts obtained through two extraction techniques: mechanical agitation and ultrasonic bath. Powdered olive leaves and a mixture of ethanol plus water (70:30) were added to a jacketed glass vessel together in a proportion 1: 3. Each experiment was performed in triplicate at temperatures from 20 to $60{ }^{\circ} \mathrm{C}$. After an extraction period of $2 \mathrm{hrs}$, the system was filtered and the supernatant was placed in a rotary evaporator to remove the ethanol and then placed into a lyophilizer for water removal. The dried extracts were analyzed by high performance liquid chromatography for oleuropein determination. Values in the range 14-16\% of oleuropein were found in the extracts. For the experiments using mechanical agitation, the oleuropein yields varied from 7 to 10\%, and those using ultrasound bath yields ranged from 6 to 9\%. The results were evaluated statistically using the Duncan test at 95\% of confidence level, using a SAS ${ }^{\circledR}$ software. Results indicated that the increase of temperature has no significant effect on oleuropein yield. It was also noted that
\end{abstract}


higher yield values were obtained using mechanical agitation; however, these results were not statistically different. This study allowed the preliminary assessment of oleuropein extraction using simple methods, mild temperatures and a GRAS solvent.

\title{
Computational Performance of Two Convergence Methods Applied to the Estimation of Viscosities for Essential Oils Industry
}

\author{
Cintia B. Gonçalves ${ }^{1, \bullet, ~ ¿, ~ C a m i l a ~ N . ~ P i n t o ~}{ }^{1}$, Gustavo V. Von Atzingen ${ }^{2}$, Priscila M. Florido ${ }^{1}$ and Christianne E. C. \\ Rodrigues $^{1}$ \\ ${ }^{1}$ University of São Paulo - FZEA/USP, Brazil \\ ${ }^{2}$ Federal Institute of São Paulo, Brazil
}

\begin{abstract}
Essential oils are mainly composed of terpenes, which are unstable to heat, and oxygenate compounds, responsible for their characteristic aroma. Determination of essential oils results in a better quality product and can be performed using solvent extraction. In this process, viscosity is important as it affects the loss of energy by friction and the mechanisms of heat and mass transfer. As viscosity can be affected by a large amount of variables, the use of appropriate methods for estimating it becomes a useful tool. The aim of this work was to study the influence of the convergence method on the modeling procedure to calculate viscosities of phases arose from the Determination of essential oils, using the UNIFAC-VISCO model. Interaction group parameters were obtained by correlating the model with experimental data of systems containing terpenes, oxygenates compounds and alcoholic solutions. The matrix of parameters obtained was used to predict viscosities of similar mixtures that were not included in the modeling. The model was programmed using MATLAB ${ }^{\circledR}$ platform and gradient descent as convergence method. Results were compared to those obtained in our previous work* using a genetic algorithm. Averages relative deviations obtained in this work for modeling and prediction ( $1.04 \%$ and $3.71 \%$, respectively) were higher than those obtained in our previous work ( $0.68 \%$ and $1.83 \%$, respectively). The modeling results show that by using the gradient descent presented a lower predictive capacity. Furthermore, interaction parameters were obtained one hundred times slower than those ones using genetic algorithm.
\end{abstract}

\section{Calculation of Viscosities of Blends Formed by Triacylglycerols, Fatty Acids and Short Chain Alcohols}

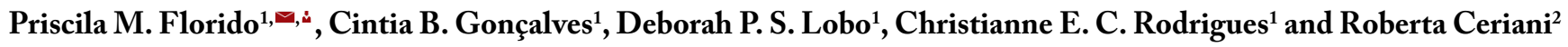 \\ ${ }^{1}$ University of São Paulo - FZEA/USP, Brazil \\ ${ }^{2}$ University of Campinas, Brazil
}

\begin{abstract}
Viscosity data allow the improvement of procedures for extracting and purifying edible oils using solvents. Due to the large number of variables that affect the process, data acquisition in all conditions of interest is not viable and methods to estimate them are fundamental. The aim of this study was to test different predictive models (Simple Kay's Rule, Modified Kay's Rule, and GC-UNIMOD) for computing viscosities of systems formed by triacylglycerols (Nu-check, purity of $99 \%)$, fatty acids (Nucheck, purity of 99\%) and short chain alcohols (Merck, purity of 99\%), which are the main components present in edible oils processing. Mixtures were prepared gravimetrically on an analytical balance, and the viscosities were measured in an Automatic Microviscosimeter (AMVn, Anton Paar). All measurements were performed in quadruplicate, with standard deviations not higher than $0.020 \mathrm{mPa}$.s. The results showed that despite the complexity of the mixtures, the simplest models (Simple and Modified Kay's Rule) were able to make a good prediction of viscosity, especially for mixtures with higher concentrations of solvent. However, for mixtures containing high concentrations of fatty compounds (triacylglycerols and fatty acids), obtaining a more effective method is still a challenge, even using a more complex model as GC-UNIMOD. Therefore, the data obtained in this work can be a starting point for the development of new models that allow a better description of this physical property, making it an important tool in the design of equipment for the industry of oils and fats.
\end{abstract}




\title{
Determination of Copper Chlorophyll in Processed Food by HPLC
}

\author{
Jae Sung Kim, Yoon Jung Yoo, Byeong Hyeon So, Kwang Sik Jung, Hyun Mi Koo, Ji Won Lee, Jang-Hyuk Ahn ־, «, Sang \\ Rag Lee and Young Ryul Kim
}

KOTITI Testing \& Research Institute, Republic of Korea

\begin{abstract}
Quantitative analytical method for copper chlorophyll in processed food was developed by using rapid sample pretreatment and HPLC-PDA (High Performance Liquid Chromatography-Photodiode Array Detector). Among various copper chlorophyll form, $\mathrm{Cu}$ (II) pyropheophytin a as a standard form was separated and validated for the analytical method. Since quantitative analytical method for the copper chlorophyll in processed foods was not published officially yet, this research was performed for the aim of official method. For the sample pre-treatment, solvent extraction was performed for removing interference effecting fats and centrifugation with weak acid was performed for proteins removal. Silica cartridge was used to capture the colorant and to remove residual fats. Reversed-phase C18 column ( $5 \mathrm{um}, 4.6$ x $250 \mathrm{~mm}$ ) to separate the colorant within 30 mins was used. Calibration curve showed good linearity with 0.9999 regression coefficients and the result of recovery test showed good recoveries within 90 110\%.
\end{abstract}

\section{Antimutagenic and Antioxidative Effects of Compound IH-901}

\author{
Jeong-Seob Park ${ }^{1, \varpi,\lrcorner, ~ E u n-B i ~ O h}{ }^{2}$ and Dong-Seong Choi $^{2}$ \\ ${ }^{1}$ Daedoo Foods Co., LTD, South Korea \\ ${ }^{2}$ Woosuk University, South Korea
}

\begin{abstract}
The compound IH 901[20-O-( $\beta$-D-glucopyranosyl)-20(S)-protopanaxadiol, compound $\mathrm{K}]$ is the main metabolite of the protopanaxadiol type of ginseng saponin produced by intestinal bacteria after oral administration of ginseng extract. In the present study, we tested the antimutagenic and antioxidative activity of the compound IH 901 on the mutagenicity induced by 10 kinds of mutagen in Salmonella typhimurium TA98 and TA100. The Compound IH 901 inhibited the mutagenicity of 4-NQO and MNNG, AFB1, Trp-P-1, Daunomycin by 65.2\%, 68.2\%, 39.7\%, and $16.1 \%$ at the concentration of $10 \mu \mathrm{g} /$ plate. The compound IH 901 decreased the reverse mutation induced by $\mathrm{H}_{2} \mathrm{O}_{2}$ and t-butyl alcohol in Salmonella typhimurium TA 102. The compound IH 901 showed strong antimutagenic effects in a dose-dependent manner. We evaluated the bioantimutagenicity of the compound IH 901 on the mutagenicity induced by 4-NQO and MNNG in Salmonella typhimurium TA100. The compound IH 901 showed time-dependent antimutagenic effects to be treated Salmonella typhimurium TA100 with 4-NQO and MNNG for 20 mins, but cell viability decreased.
\end{abstract}

\section{Antimutagenic Activity of Falcarinol Isolated from Eleutherococcus senticosus Maxim. Cultivated in South Korea}

\author{
Dong-Seong Choi ${ }^{1, ๘,\lrcorner}$,Jeong-Seob Park ${ }^{2}$, Eun-Bi Oh ${ }^{1}$ and Young-Hoi Kim ${ }^{3}$ \\ ${ }^{1}$ Woosuk University, South Korea \\ ${ }^{2}$ Daedoo Foods Co., LTD, South Korea \\ ${ }^{3}$ Chonbuk National University, South Korea
}

\begin{abstract}
In the present study, we tested antimutagenic effect of Eleutherococcus senticosus Maxim. Root (ESM) on the mutagenicity induced by 2-aminofluorene and Trp-P-1 in Salmonella typhimurium TA98. Crude methanolic ESM extract showed the strong antimutagenic activity. The methanol extract of ESM was further fractionated to chloroform, butanol, and water fractions. The chloroform fraction showed a suppressive effect against the mutagenicity of Salmonella typhimurium TA98. Two compounds (compound I and II) were isolated from chloroform fraction by silica gel column chromatography and identified by GC-MS, $1 \mathrm{H}$ and 13C NMR spectroscopy. The composition of compound I was confirmed to be a mixture of falcarinol, hinokinin, phthalate derivatives and $\alpha$-bisabolene. The compound II was found to be a mixture of hinokinin, savinin, savinin derivatives and helioxanthin. Among them, falcarinol was further isolated and purified. The falcarinol showed strong antimutagenic effects in a dose-dependent manner. In addition, the falcarinol showed suppressive effect on the Ames test in Salmonella typhimurium TA98 and TA100 against 1-nitropyrene (1-NP) induced mutagenicity.
\end{abstract}




\title{
Antioxidative and Antimutagenic Effects of Proso Millet
}

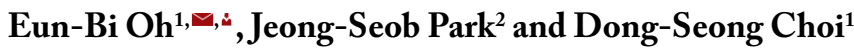 \\ ${ }^{1}$ Woosuk University, South Korea \\ ${ }^{2}$ Daedoo Foods Co., LTD, South Korea
}

\begin{abstract}
Antioxidative and antimutagenic effects of the $80 \%$ methanol extract of Prose millet were examined by DPPH radical scavenging activity and total polyphenol content, and Ames test using Salmonella. The DPPH radical scavenging activity (EC50) and total polyphenol of 80\% methanol extract of Prose millet were $67.43 \mathrm{mg} / \mathrm{wt}$ and $16.26 \mathrm{mg} / \mathrm{wt}$, respectively. The $80 \%$ methanol extracts were further fractionated into chloroform, n-butanol and water fractions. The chloroform fraction showed the strong antioxidative effects as shown in DPPH radical scavenging activity and total polyphenol content. The antimutagenic effects of Prose millet toward aflatoxin B1 (AFB1), Trp-P-1, 2-aminofluorence (2-AF) induced mutagenicities using the Ames assay system with Salmonella typhimurium TA98 and TA100 were studied. The $80 \%$ methanol extracts (400 $\mu \mathrm{g} / \mathrm{plate})$ exerted the 98.3, 89.5, 97.2\% inhibitory effects against AFB1, Trp-P-1, 2-AF induced S. typhimurium TA98 mutagenicity and the 93.3, 49.2\% inhibitory effects against AFB1, 2-AF induced $S$. typhimurium TA100 mutagenicity, respectively. The chloroform fraction showed the most strong antimutagenic effects on the mutagenicity induced by AFB1, Trp-P-1, 2-AF in S. typhimurium TA98 and TA100, dose-dependently.
\end{abstract}

\section{Cis/Trans Isomerization of Cholecalciferol in Microencapsulated Vitamin D3}

\author{
Fatemeh Mahmoodani ${ }^{1, \bullet, \varkappa}$, Conrad Perera ${ }^{1}$, Grant Abernethy ${ }^{2}$, Bruno Fedrizzi $^{1}$ and Hong Chen $^{2}$ \\ ${ }^{1}$ University of Auckland, New Zealand \\ ${ }^{2}$ Fonterra Cooperative Group Ltd, New Zealand
}

\begin{abstract}
Vitamin D (VD) fortification has been in the centre of attention in recent times, because VD insufficiency is commonly found in many populations worldwide and that it has an effect on many physiological systems in the body. Therefore, the stability of VD added is important. VD is known to isomerise under various conditions, thereby making its stability in fortified products uncertain and its analysis challenging. This paper reports the cis/trans isomerization of microencapsulated VD3 by thermal and iodine treatment. The reaction of VD3 and pre-VD3 in non-polar solvents with a small amount of iodine yields two different isomers. While VD3 was thermally and reversibly transformed to pre-VD3, the Trans form of pre-VD3 is irreversibly formed in the presence of iodine. VD3 is usually microencapsulated in a carbohydrate matrix before adding into products to stabilize it. Efficient methods to dissolve this carbohydrate matrix and to extract the nutrient are important for its analysis. To do so, the microencapsulated VD3 was dissolved in a 10\% (v/v) aqueous DMSO solution and $20 \mathrm{~mL}$ of hexane was added, mixed well and centrifuged. The supernatant was analysed using a reversed-phase HPLC with a Kinetex PFP column to provide a good separation and efficiency. The spectra of two isomers support the view that they should be considered as Trans isomers of VD3 and pre-VD3. The results may provide potential degradation pathways of cholecalciferol in microencapsulated VD3 and useful information to VD3 chemistry.
\end{abstract}

\section{Characterization of Phenolic Compounds as Potential Markers for the Floral Origin of New Zealand Unifloral Honeys}

\author{
Zahra Zolfaghari" ${ }^{\varpi, ¿, ~ G r a h a m ~ E y r e s ~ a n d ~ J o h n ~ B i r c h ~}$ \\ University of Otago, New Zealand
}

\begin{abstract}
In this study the phenolic profiles and antioxidant activity of New Zealand unifloral honey samples (manuka, kamahi, rata, clover and thyme) were determined. The extraction of phenolic compounds was performed using Amberlite-XAD-2 as a solidphase extraction sorbent. The composition of the honey extracts were analysed using HPLC with photodiode array detector and the identification of phenolics was performed by comparison of their retention times with those of external standards solutions. Total phenol contents, DPPH and FRAP assays were also applied to investigate the antioxidant activity of honey samples. The obtained results confirmed that the composition of phenolic fractions extracted from honeys is highly influenced by their floral
\end{abstract}


origin and could be applied as possible markers of their botanical origin. Antioxidant activity tests showed that thyme and clover honey had the highest and lowest antioxidant activity, respectively.

\title{
Effect of Stevia Sweetener Consumption as Low Calorie Sweetening on Overweight and Obesity in Female Rats
}

\author{
Abo Elnaga NIE ${ }^{1, \varpi, ¿, ~ M o n a ~ I . ~ M a s s o u d ~}{ }^{2}$, Yousef MI ${ }^{1}$ and Mouhamed HHA ${ }^{1}$ \\ ${ }^{1}$ Alexandria University, Egypt \\ ${ }^{2}$ Sugar Crops Research Institute, Egypt
}

\begin{abstract}
Recently, the prevalence of overweight children and adults has increased and diet choices affect weight gain. Non-caloric or low caloric sweeteners as tools for making healthy food choices have been introduced to satisfy consumer demand. The aim of this study was to evaluate the effect of stevia sweetener as a substitute sucrose at different doses $(25,250,500$ and $1000 \mathrm{mg} / \mathrm{kg}$ b. wt./day) for twelve weeks on the weight management and on several hematological and biochemical parameters of female rats. The results showed significantly improvement and ameliorated reduction in final bodyweight, body weights gain (BWG \%) and feed efficiency ratio (FER) in the stevia sweetener groups compared with the control. Stevia sweetener at $500 \mathrm{mg} / \mathrm{kg}$ b.wt/day help's in weight loss of rats, decrement in the total cholesterol, triglycerides and low-density lipoprotein (LDL) concentration, increment in the high-density lipoprotein (HDL) and no significant differences in mean serum alanine transaminase (ALT), aspartate transaminase (AST), alkaline phosphatase (ALP), or acid phosphatase (ACP) levels as compared to the control rats, which may be considered as therapeutic beneficial. Also, this dose may be considered as a safe dose for people with diabetes, especially the many individuals who need to lose weight to help control their blood glucose levels.
\end{abstract}

\section{Exposure Assessment of Food Preservative in the Total Diet in Taiwan}

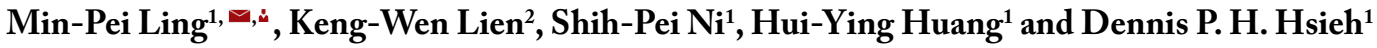 \\ ${ }^{1}$ China Medical University, Taiwan \\ ${ }^{2}$ National Taiwan University, Taiwan
}

\begin{abstract}
The purpose was to assess the health risk to general consumers in Taiwan associated with dietary intake of benzoic acid and sorbic acid by conducting a total diet study (TDS). The hazard index (HI) in percent acceptable daily intake (\%ADI) of benzoic acid and sorbic acid for eight exposure groups classified by age were calculated. In high-intake consumers, the highest HI of benzoic acid was 54.1\% ADI for males aged 1-2 years old at the $95^{\text {th }}$ percentile, whereas for females, the HI was $61.7 \%$ ADI for aged over 66 years old. The highest HI of sorbic acid for male and female consumers aged 3-6 years old at the $95^{\text {th }}$ percentile were $14.0 \% \mathrm{ADI}$ and $12.2 \% \mathrm{ADI}$, respectively. These results indicate that the use of benzoic acid and sorbic acid as preservatives at the current level of use in the Taiwanese diet does not constitute a public health and safety concern.
\end{abstract}

\section{Improvement of the Method for the Determination of Ki-Value by Using Amorphous Freeze-Dried Enzymes}

\author{
Hiroko Seki $₫$,^, Izumi Ueno, Toru Suzuki and Naoko Hamada-Sato \\ Tokyo University of marine science and technology, Japan
}

\begin{abstract}
The Ki-value is an approximation of the $\mathrm{K}$-value used for determining the freshness of fish. A method for rapidly and easily determining freshness using the Ki-value has been developed previously in our laboratory. Itconsists of application A that includes the use of xanthine oxidase (XOD) and nucleoside phosphorylase (NP) to determine the amount of inosine (HxR) and hypoxanthine (Hx), and application B that includes XOD, NP, and alkaline phosphatase (ALP) to determine the amount of inosinic acid (IMP), HxR, and Hx. Both applications use a coupler (WST-8), and Ki-value is calculated by colorimetry. However, the overall reaction time in this method is about 30 mins. Therefore, we revisited our method with an aim to shorten the reaction time. We also focused on evaluating the quality of not only fish but also pork and compared the Ki-value obtained
\end{abstract}


using the improved method developed in this study with the K-value obtained using High performance liquid chromatography (HPLC). We aimed to shorten the reaction time by using different enzyme concentrations in both applications. Using the following concentrations of XOD, NP,WST-8, and ALP, we succeeded in reducing the reaction time to 10 mins: XOD 0.6 U/ $\mathrm{mL}, \mathrm{NP}$ 0.6 U/mL, $1.2 \mathrm{mM}$ WST-8in application A, and XOD 0.6 U/mL, NP 0.6 U/mL, ALP $40 \mathrm{U} / \mathrm{mL}$, and $1.2 \mathrm{mM} \mathrm{WST}$ 8 in application B. Moreover, we confirmed a high correlation between Ki-value and K-value in pork as well. Thus, the improved method developed here can be applied in the evaluation pork quality.

\title{
The Type II RIP Aralin from Aralia elatais Preferentially Incorporated into Tumor Cells via the High Density Lipoprotein-Binding Protein-Dependent Endocytosis and Induces Cell Death
}

\author{
Hiroko Otsuka ${ }^{1, \bullet,\llcorner, ~ Y o s h i t a k a ~ G o t o h ~}{ }^{1}$, Takashi Komeno ${ }^{1}$, Takahide Ono ${ }^{1}$, Yasushi Kawasaki ${ }^{1}$, Naoyuki Iida ${ }^{2}$, Yoshio \\ Shibagaki $^{2}$, Seisuke Hattori ${ }^{2}$, Makoto Tomatsu ${ }^{3}$, Hirotada Akiyama ${ }^{1}$ and Fumio Tashiro ${ }^{1}$ \\ ${ }^{1}$ Tokyo University of Science, Japan \\ ${ }^{2}$ Kitasato University, Japan \\ ${ }^{3}$ Akita Research Institute of Food and Brewing (ARIF), Japan
}

\begin{abstract}
Aralin from Aralia elata is a newly identified type II ribosome inactivating protein, which preferentially induces apoptosis in cancer cells. However, the mechanisms underlying the selective cytotoxicity of aralin for cancer cells remain obscure. Here, we report that aralin enters the cell by first binding to the $110-\mathrm{kDa}$ high-density lipoprotein-binding protein (HDLBP) as a cell surface receptor, then crossing the plasma membrane via endocytosis and finally invading the cytosol via translocation from the Golgi apparatus, endoplasmic reticulum (ER)-Golgi intermediate compartment (ERGIC)-53 and ER. The sensitivities of tumor cell lines to aralin were dependent on the expression levels of the 110-kDa HDLBP and its forced expression in aralin-resistant Huh7 cells conferred aralin sensitivity. HDLBP-knockdown HeLa cells showed a significant aralin resistance in vitro and in vivo. Conversely, ectopic expression of the $150-\mathrm{kDa}$ HDLBP resulted in increased aralin sensitivity in vivo, accompanying enhanced expression of the $110-\mathrm{kDa}$ HDLBP. Thus, these results showed that the110-kDa HDLBP in lipid rafts acted as an aralin receptor and that its expression levels determined aralin sensitivity, suggesting that aralin could be a promising anticancer drug for HDLBP-over expressing tumors. This research was supported by a grant (15162MFDS039) from Ministry of Food and Drug Safety in 2015.
\end{abstract}

\section{Simultaneous Determination of Ten Photo Initiators in Milk by Solid-Phase Micro Extraction Coupled with Gas Chromatography/Mass Spectrometry}

\author{
Pengyan Liu ${ }^{\complement, \varkappa}$, Yanjie Chen, Chunxia Zhao, Yajing Zhang, Ruirui Zhang and Yana Zhang \\ Hebei University, China
}

\begin{abstract}
Photoinitiators (PIs), an important part of the ultraviolet(UV) curing ink, are widelyused in food packaging materials like paper and plastics. Most of them can migrate easily from packaging materials to food, causing food contamination. Establishing method for simultaneous determination of multi-residues PIs in food has important practical significance. A new method for simultaneously determining ten kinds of PIs, benzophenone (BP), ethyl 4-dimethylaminobenzoate(EDMAB), 1-hydroxycyclohexylphenylketone(CPK), 4-methylbenzo-phenone(4-MBP), 2-ethylhexyl-4-dimethylaminobenzoate(EHDAB), 4-chlorobenzophenone(p-CBP),2-chlorothioxanthone(CTX),2-isopropylthioxanthone(ITX),2,2-dimethoxy-2-phenyl-acetophenone(2,2DMPA), methyl 2-benzoylbenzoate(OMBB),in milk has been established by using solid-phase micro-extraction (SPME) combined with a simple method of protein precipitation as the pretreatment approach and GC/MS as the detecting technique. The major factors affecting SPME, extraction time and temperature, were studied and the optimal operation conditions were found through factor experiment. The working curves were obtained using milk samples with different fat contents. The linear range of six kinds of PIs (BP, EDMAB, 4-MBP, P-CBP, 2, 2-DMPA, OMBB) were from $2.0 \mu \mathrm{g} / \mathrm{L}$ to $200 \mu \mathrm{g} / \mathrm{L}$ and four kinds of PIs(CPK, EHDAB, CTX, and ITX) were from $10 \mu \mathrm{g} / \mathrm{L}$ to $1000 \mu \mathrm{g} / \mathrm{L}$. In skimmed milk, the limits of detection were between $0.05 \mu \mathrm{g} / \mathrm{L}$ and $1.4 \mu \mathrm{g} / \mathrm{L}$. The samples were determined three times with three different spiked levels respectively and the relative standard deviations of all the samples were less than $14.1 \%$. Three positive samples were found in 12 kinds of packed milk with different brands and fat contents using this determination method.
\end{abstract}




\title{
Three Bioactive Compounds in Citrus aurantifolia Peel Inhibits Adipogenesis with Different Degree of Potencies, A Potential Source for Anti-Obesity Therapeutics
}

\author{
Asyifah Mohamed Rashid ${ }^{\bullet, \gtrsim}$, Yew Mun Yip and Dawei Zhang \\ Nanyang Technological University, Singapore
}

\begin{abstract}
Citrus aurantifolia, commonly known as lime or limaunipis, has been used traditionally, over centuries long, to treat various sickness. In this study, we focused on the peel component of Citrus Aurantifolia and it has been found for the first time, that the crude lime peel extract (LPE) was able to significantly inhibit adipogenesis in a dose-dependent manner. Upon further purification and isolation of the crude extract, we discovered that it is the presence of three poly methoxylated flavones (PMFs), particularly Nobiletin, Tangeretin and Sinensetin in LPE that accounts for its bioactivity. In addition, our study further revealed that the three PMFs, although structurally similar, exhibit different potencies in adipocyte differentiation and this is attributed to the differing binding interactions that Nobiletin, Tangeretin and Sinensetin has with PPAR $\alpha$ that accounts for the difference in the degree of up regulation of PPAR $\alpha$ gene expression during adipocyte differentiation. Fundamentally, the ability of the three bioactive compounds elucidated in this study to effectively inhibit adipocyte differentiation further supports the potential of lime peel extract as a prospective source of supplement to treat obesity.
\end{abstract}

\section{The Effect of Storage Temperature on the Crystalline and Resistant Starch Properties of White Bread}

\author{
William R. Sullivan ${ }^{\square, \varkappa}$, Russell W. Cockman and Darryl M. Small \\ Royal Melbourne Institute of Technology (RMIT) University, Australia
}

\begin{abstract}
Resistant starch (RS) is the portion of starch that we are unable to readily digested. RS has the ability to act as a carbon source for friendly bacteria within the colon resulting in a variety of health benefits including a decreased risk of bowel cancer as well as weight management. The purpose of the current study has been to investigate changes in RS content and starch crystallinity of bread during storage at different temperatures. Loaves of white bread were baked and stored at refrigeration, frozen and room temperatures with analysis on days $0,1,4$ and 7 . Analyses have included RS determination using an enzymatic procedure and X-ray diffraction to evaluate the relationship between total crystallinity and the RS content, as influenced by storage temperature. Based on the diffractogram patterns it was found that the slowest recrystallization occurred in the frozen bread, samples followed by the refrigerated bread and then the room temperature samples.
\end{abstract}

\section{The Influence of Heat Treatments on Cereal Starches}

\author{
Sana Subzwari ${ }^{1, œ, \Perp, ~ W i l l ~ S u l l i v a n ~}{ }^{1}$, Adel Fickak $^{2}$, Russell Cockman $^{1}$ and Darryl Small $^{1}$ \\ ${ }^{1}$ RMIT University, Australia \\ ${ }^{2}$ Monash University, Australia
}

\begin{abstract}
Starches from cereal grains vary in their granular, compositional and many other properties. In the context of studies designed to enhance our understanding of these granules and their application as encapsulating agents, the effects of heating during spray drying have been investigated. Starches have been treated by producing powders, using a Niro Atomiser laboratory instrument. Granules were suspended in water to form a slurry using gentle stirring. The effects of spray drying conditions, inlet air temperature $\left(80^{\circ} \mathrm{C}\right.$ and $200{ }^{\circ} \mathrm{C}$ ) and outlet temperatures $\left(80,120,150\right.$ and $200{ }^{\circ} \mathrm{C}$ ) at a flow rate of $15 \mathrm{~mL} / \mathrm{min}$ have been evaluated. The moisture content, particle size distribution and crystallinity were evaluated using oven drying, laser scattering and $\mathrm{X}$-ray diffraction, respectively. The outlet temperature was found to strongly affect the moisture content and physical changes to both the periphery and through the hilum were observed by ESEM and in X-ray patterns. Spray-drying conditions involving an outlet temperature of $120-150{ }^{\circ} \mathrm{C}$ and a flow rate of $15 \mathrm{~mL} / \mathrm{min}$ gave a moisture content of approximately $8 \%$. In addition, no changes in granular size were observed. The implications of the findings warrant further analysis of resistant starch and an extended range of cereal starches.
\end{abstract}




\title{
Microbial and Biochemical Assessment of Fresh Date (Phoenix dactylifera L.) Fruits
}

\author{
F. Al-asmari ${ }^{1,2, モ, 2}$, N. Nirmal ${ }^{1}$, M. Chaliha ${ }^{1}$, D. Williams ${ }^{3}$, R. Mereddy $^{3}$, K. Shelat ${ }^{4}$ and Y. Sultanbawa $^{1}$ \\ ${ }^{1}$ Queensland Alliance for Agricultural and Food Innovation (QAAFI), TheUniversity of Queensland, Australia \\ ${ }^{2}$ King Faisal University, Saudi Arabia \\ ${ }^{3}$ Department of Agriculture andFisheries Queensland, Australia \\ ${ }^{4}$ Australian Institute of Nano and Bioengineering (AIBN), Australian National Fabrication Facility (ANFF), The University of \\ Queensland, Australia
}

\begin{abstract}
Fungal contamination plays a significant role in spoilage of pre and post-harvest date fruits. The present study aimed to identify the fungal populations and possible toxins associated with four date varieties (Sukary, Birhi, Khlass and Rothan) collected from different regions in Saudi Arabia. Biochemical characteristics including moisture, $\mathrm{pH}$, sugar content, total phenolic content, radical scavenging activity and mycotoxin levels were determined. The moisture and $\mathrm{pH}$ of the dates ranged from $37.8 \%$ to $58.4 \%$ and $5.3-6.8$, respectively in the four varieties. Total phenolic content ranged from 148 to $242 \mathrm{mg}$ $\mathrm{GAE} / \mathrm{g}$ for the fresh dates. The major sugars glucose, fructose and sucrose were determined colorimetrically Mycotoxins were not detected in any of the tested dates. Microbial assessment revealed, mesophilic aerobic bacteria $<1000 \mathrm{cfu} / \mathrm{mL}$, moulds and yeasts $<100 \mathrm{cfu} / \mathrm{mL}$, and coliforms $<10 \mathrm{cfu} / \mathrm{mL}$. Fifteen fungal species belonging to ten genera were isolated and identified by the gene sequencing method. Aspergillus sp., Rhizopus sp., Altenrnaria sp. and Penicillium sp. were found to be the dominant genera. The most frequent fungal species were $A$. niger $100 \%, A$. ustus $77.5 \%$ R. stolonifer and Sarocladium implicatum $62.5 \%$ Altenrnaria sp. $27.5 \%$ and Penicillum sp. $25 \%$. The highest total fungal contamination was observed in the Birhi with $36 \%$ followed by Khlass $33 \%$, Sukary $29 \%$ and Rothana 19\%. The details of these biochemical analysis and impact on post-harvest quality of these dates will be discussed.
\end{abstract}

\section{Characterization of Poly (Propylene Carbonate), Sustainable Polymer for Food Packaging Applications}

\author{
Bahareh Bahramian ${ }^{\complement,\llcorner a n d ~ F a r i b a ~ D e h g h a n i ~}$ \\ University of Sydney, Australia
}

\begin{abstract}
Disposal of non-degradable polymeric wastes is a major issue due to the limited availability of landfill sites and their negative impacts on the environment and ecosystems. Around $40 \%$ of the annual plastic wastes, disposed to the landfills, are form food packaging materials. Development of a new generation of degradable and eco-friendly plastics can be a promising approach to address such environmental issues. Poly (propylene carbonate) (PPC) is a biodegradable and partially renewable polymer that is synthesized from copolymerization of $\mathrm{CO}_{2}$ and propylene oxide (PO). In the present study PPC was fully characterized and its potential to alter commercial non-degradable polymers for food packaging applications was investigated. The results demonstrated that tensile modulus of PPC was three-fold more than biodegradable Ecoflex and comparable with non-degradable low density polyethylene (LDPE) that are commercially used for food packaging. Furthermore, tear resistance of PPC was found to be five-times higher than LDPE. In terms of barrier properties, the results of oxygen transition rate (OTR)and water vapor transition rate (WVTR) measurements exhibited remarkably higher resistance to oxygen and water vapor permeation for PPC comparing to other commercial polymers. High barrier properties are one of the critical factors for food packaging that inhibits food spoilage and extend the shelf-life of packaged food. Furthermore, PPC was found to be chemically resistant and stable in various food simulated media. In summary, we demonstrate that PPC has superior properties and can be contemplated as an alternative to polyethylene and other polymers for food packaging to reduce the environmental impact of non-degradable polymers.
\end{abstract}

\section{Effect of Different Drying Methods on Sweetener Capacity and Chemical Composition of Stevia rebaudiana Leaves}

\author{
Antonio Vega-Galvez ${ }^{1, \varpi, ょ, ~ R o b e r t o ~ L e m u s-M o n d a c a ~}{ }^{1}$, Elsa Uribe, Pilar Rojas ${ }^{1}$, Luis Puente $^{2}$ and Carla Delporte $^{2}$ \\ ${ }^{1}$ Universidad de La Serena, Chile \\ ${ }^{2}$ Universidad de Chile, Chile
}




\begin{abstract}
Stevia has been used as a natural sweetener in some Latin-American countries since centuries and nowadays the consumption of this leaves is growing around the world. Sweetener obtained from leaves is 300 times sweeter than sucrose, with no contribution of calories and do not produce changes in sugar levels in blood torrent. The aim of this work was to use different drying treatments: freeze drying, forced air, vacuum drying, microwaves, infra-red radiation and open sun conditions in Stevia rebaudiana leaves in order to study the effect of these treatments on chemical composition, sweetener content and antioxidant compounds. Regarding to chemical composition of stevia leaves have a high content of insoluble fiber $(29.5 \%$ $31.4 \%), \alpha$-linolenic acid (1.36 - 5.96\%), vitamin C and E (123 and $15.3 \mathrm{mg} / 100)$ respectively, vacuum drying and freeze drying were the methods with best results in maintaining chemical composition. Steviosid was the main sweet glucoside found in leaves and forced air yield the best results. Freeze drying gives the best results in keeping antioxidant compounds in high levels. As conclusion all the used methods were useful in stevia leaves processing, but it depends on the final use of the dried leaves (sweetener, antioxidant or as an ingredient).
\end{abstract}

\title{
Different Drying Methods to Murta (Ugni molinae Turcz) Fruit Berries: Changes in Phenolic Compounds and Antioxidant Activity
}

\author{
Antonio Vega-Gálvez ${ }^{1,2, \varpi, ¿ ~}$, Jéssica López $^{1}$, Elsa Uribe ${ }^{1}$ and Luis Puente-Díaz ${ }^{3}$ \\ ${ }^{1}$ Department of Food Engineering, Universidad de La Serena, Chile \\ ${ }^{2}$ CEAZA, Center for Advanced Studies in Arid Zones, Universidad de La Serena, Chile \\ ${ }^{3}$ Universidad de Chile, Chile
}

\begin{abstract}
Ugni molinae Turcz, commonly known as "murtilla, "murta" or "Chilean guava" is a Chilean native shrub native growing wildly in the southern regions of Chile. It belongs to the Myrtaceae family and this plant produces a small globoid berry (Alfaro et al., 2013). This fruit is produced on a seasonal basis from March to May, and drying is an adequate technique for its preservation. Therefore, the aim of this study was to study the effect of three different drying methods on the flavonoid content, phenolic profile and antioxidant activity in murta berries. The methods studied were convective drying (CD), vacuum drying (VD) and freeze drying (FD). High-performance liquid chromatography (HPLC) coupled with diode-array detection was used to identify and quantify the phenolic compounds and the antioxidant capacity was determined by ORAC assay. The results showed that the dehydrated murta presented a profile composed of: flavonoids (Rutin hydrate and Quercetin) and phenolic acids (gallic, ellagic, and trans-cinnamic acids). The data shows that rutin hydrate was the predominant flavonoid in the dried samples. Rutin Hydrate showed significant decrease applying drying methods. All the drying processes affected the retention of phenolic compounds. Where Gallic and ellagic acids showed an increase compared to the fresh sample especially in FD. The CD caused a decrease of $61 \%$ in antioxidant activity whereas the FD caused a decrease of $20 \%$. This study has demonstrated the ideal method for drying was FD, being the ones that maintained more the antioxidant activity and is good for retaining phenolic compounds.
\end{abstract}

\section{Effect of a Commercial Enzyme on the Diacetyl Content during the Fermentation and Maturation of Lager Beer}

\author{
Fernando Salazar ${ }^{1, \varpi, ょ, ~ M a r i e l a ~ L a b b e ́ ~}{ }^{1}$, Michelle Carrasco ${ }^{1}$, Pablo Ulloa $^{1}$ and Francisco López ${ }^{2}$ \\ ${ }^{1}$ Pontificia Universidad Católica de Valparaíso, Chile \\ ${ }^{2}$ Universitat Rovirai Virgili, Spain
}

\begin{abstract}
Diacetyl (2, 3 butanedione) and 2, 3-pentanedione are vicinal diketones (VDK) formed during wort fermentation as byproducts of yeast metabolism. The presence of diacetyl is undesirable because it produces a butter-tasting in beer which can be reduced by maturation time. Reducing concentrations of diacetyl in green beer represents an expensive and time-consuming part of the brewing process. Therefore, strategies to minimize diacetyl formation or hasten its reduction are required for improving overall efficiency of the lager brewing system. In this study four industrial lager brewing trials of $12 \mathrm{hL}$ were made by duplicate. A control and three different doses $(0.8,1.2$ and $2 \mathrm{~g} / \mathrm{hL})$ of a commercial $\alpha$-acetolactedescarboxylase enzyme (Maturex $\left.{ }^{\circledR}\right)$ were added at the beginning of the fermentation using Saccharomyces cerevisiae strain w34/70 (Fermentis ${ }^{\circledR}$ ). Diacetyl content was monitored during the fermentation and maturation of treated and non-treated wort by enzyme. Free amino nitrogen in
\end{abstract}


wort and yeast viability at the end of fermentation were controlled in order to minimize the effect of these parameters on the diacetyl formation. The addition of enzyme during the wort fermentation reduced the diacetyl content to a level below the taste threshold without affecting other important sensory properties of the beer. Consequently, it is concluded, that application of $\alpha$-acetolactedescarboxylase may allow flavor maturation of beer to be carried out in less time than a conventional prepared beer without enzyme addition.

\title{
Active Bio-Packaging with Propolis as Active Agent
}

\author{
Pablo A. Ulloa ${ }^{1, \bullet, ¿}$, Juan Vidal ${ }^{1}$, Fernando Salazar ${ }^{1}$, Abel Guarda $^{2}$ and Maria Jose Galotto ${ }^{2}$ \\ ${ }^{1}$ Pontificia Universidad Católica de Valparaíso, Chile \\ ${ }^{2}$ Universidad de Santiago de Chile, Chile
}

\begin{abstract}
The use of synthetic polymers produces large amount of solid waste and contamination to the environmental around the world. For that reason, many researchers are focused about to development of the biodegradable polymers such as poly lactic acid (PLA). PLA offers great promise in a wide range of commodity applications and competes with polystyrenes for many food-packaging applications. The goal of this study was the characterization of active films of PLA with powder raw propolis as active agent. Besides, were evaluated the release of bioactive compounds (phenol total and flavonoids total) from PLA matrix. Active bio-packaging films were obtained by "solvent cast" method and were prepared three films with different concentration (5, 8.5 and 13\% w/w PLA) of propolis. Results obtained to mechanical and optical properties for the active films (PLA+propolis) showed a decreased in the elasticity modulus, tensile strength and percentage of elongation and were detected changes of the color in comparison with PLA control (polymer without propolis). This behavior was more evident for higher propolis concentration. Degradability results shown a loss of weight about 5.4\% to PLA (control) and 3.3\% (PLA+propolis) after 168 days under soil. Results for the release of bioactive compounds evaluated at $36 \mathrm{hrs}$, showed that the release and available of these compounds were higher with PLA+propolis films with high concentration of active agent. We concluded that was possible obtained an active bio-packaging with release bioactive compounds that will have antioxidant property if used as food package.
\end{abstract}

\section{Monitoring Peroxides Generation During Model Wine Fermentation by FOX-1 Assay}

\author{
Raquel Bridi ${ }^{1, \bullet, £, ~ A l v a r o ~ G o n z a ́ l e z ², ~ E d m u n d o ~ B o r d e u ², ~ C a m i l o ~ L o ́ p e z-A l a r c o ́ n ~}{ }^{1}$, Alexis Aspée ${ }^{3}$, Benjamin Diethelm¹, \\ Eduardo Lissi ${ }^{3}$, Giuseppina Paola Parpinello ${ }^{4}$ and Andrea Versari ${ }^{4}$
}

${ }^{1}$ Pontificia Universidad Católica de Chile, Facultad de Química, Chile

${ }^{2}$ Pontificia Universidad Católica de Chile, Facultad de Agronomía e Ingeniería Forestal, Chile

${ }^{3}$ Universidad de Santiago de Chile, Chile

${ }^{4}$ University of Bologna, Italy

\begin{abstract}
The alcoholic fermentation, which gradually transforms the grape juice into wine, strongly affects the quality of wine. Along this process, several stressful stages of yeast affect its fermentative ability, therefore the industrial performance and the wine quality. In this context, the quantification of reactive oxygen species (ROS) or their related products (as hydroperoxides) can provide useful information on the ongoing of fermentation which would allow improving the prediction of wine quality. In the present work, for first time FOX-1 assay was used to determine total hydroperoxides in a model winemaking condition. For such purpose, we inoculated commercial Saccharomyces cerevisiae strains (EC1118, QA23, VIN7 and VL3) in batch laboratory fermentations employing a model wine solution. The highest hydroperoxide production was determined for strain EC1118 (peak at $10.0 \mu \mathrm{M}$ on day 5, of which $1.3 \mu \mathrm{M}$ was hydrogen peroxide) and VL3 (peak at $9.1 \mu \mathrm{M}$ on day 3), followed by strain QA23 (peak at $6.0 \mu \mathrm{M}$ on day 6) and VIN7 (peak at $5.5 \mu \mathrm{M}$ on day 6). Based on our results we conclude that the FOX-1 based analytical approach is a valuable tool for the quantification of ROS oxidative damage during the winemaking process.
\end{abstract}




\title{
Multilayer Oil-in-Water (O/W) Emulsion System Increases the Retention of Volatile Flavors during Spray-Drying
}

\author{
César Burgos-Díaz ${ }^{1, \boxminus, \varkappa}$, José A Piornos ${ }^{1}$, Xaviera Hernandez $^{2}$ and Mónica Rubilar ${ }^{1,2}$ \\ ${ }^{1}$ Agriaquaculture Nutritional Genomic Center, CGNA, Chile \\ ${ }^{2}$ Universidad de La Frontera, Chile
}

\begin{abstract}
The incorporation of hydrophobic volatile food flavor molecules into dry powder is of great interest to food industry. Spray drying is the most commonly used technique for the production of dry flavorings. However, the high temperatures used for spray drying can lead to the loss of volatile molecules. The use of multilayer emulsion as a system of encapsulation of aromas could be an alternative to improve the stability of emulsions $(\mathrm{O} / \mathrm{W})$ and thus reduce the loss of volatile aroma during food processing and subsequent storage. Therefore, the objective of this study was to evaluate the effect of the utilization of multilayer emulsions on the retention of flavor (L-limonene) during spray-drying. For this, the multilayer O/W emulsions were stabilized by using protein isolate from a novel protein-rich lupin variety, Alu Prot-CGNA (LPIA) and chitosan. The multilayer O/W emulsions, containing10 wt. \% oil, 0.5 wt. \% LPIA, 0.06 wt. \% chitosan and 1,000 ppm flavor (limonene), were prepare during the layerby-layer technique. Then, emulsions were dried at a laboratory scale spray-drier, and the total amount of flavor compounds in the powder was measured. The preliminary results showed that multilayer emulsions, using chitosan as an additional oil droplet layer, were more effective in the retention of flavor compounds than monolayer emulsions (LPIA alone). Finally, the use of multilayer emulsions, as a flavor microencapsulation system, was able to increase the retention of volatile molecules during spray-drying. These findings have important implications for the design of encapsulation and delivery systems for lipophilic compounds in the food industries.
\end{abstract}

\section{The Effect of Prickly Pear by Products on the Oxidation Stability of Margarine}

\author{
Nadia Chougui ${ }^{1, \bullet, \longleftarrow}$, Naima Djerroud ${ }^{1}$, Fatima Naraoui ${ }^{1}$, Samir Hadjal ${ }^{2}$, Khellaf Aliane ${ }^{2}$, Brahim Zeroual ${ }^{2}$ and Romain \\ Larbat $^{3,4}$ \\ ${ }^{1}$ Université de Bejaia, Algeria \\ ${ }^{2}$ Cévital spa, Algeria \\ ${ }^{3}$ INRA UMR, France \\ ${ }^{4}$ Université de Lorraine UMR, France
}

\begin{abstract}
Lipid oxidation is a major cause of degradation of margarine during its manufacture and its conservation. The most noticeable consequence is the development of unpleasant odors leading to the rejection of the product by the consumer. To prevent oxidation of margarine, industries add synthetic antioxidants such as Tocoblend (vitamin E) which is the most widely used. The trend today is to use natural substances that play the same role without changing the properties of the product. One of the most appropriate approaches is the exploitation of extracts of food waste. In this context, the study aimed to substitute, in the margarine vitamin $\mathrm{E}$, with food by-products extract. In this study, we have tested the hydro-ethanolic extract of prickly pear peels that represent 40 to $50 \%$ of the whole fruit and constitute a source of bioactive compounds. Results showed that the peel extract was indeed rich in total phenolics (1512.58 mg GAE/100 g DM). HPLC-DAD-ESI-MSn analyses allowed the identification of sixteen compounds belonging to hydroxybenzoic acids, hydroxycinnamic acids and flavonoids. The extract displayed an antioxidant activity that was similar to the two powerful antioxidant standards quercetin and butylated hydroxyanisole. Tests conducted at laboratory and pilot scales showed that the margarines elaborated with peel extract were more resistant to oxidation than the margarine reference with vitamin E. In addition, neither the physicochemical nor the microbiological properties were modified. Prickly pear peels contain bioactive substances that could be used in different food sectors.
\end{abstract}




\title{
Date Stone as Source of Phenolic Antioxidants Extracted by Microwaves: Optimization Study
}

\author{
Hayate Haddadi-Guemghar ${ }^{1, \bullet,\llcorner, ~ K e n z a ~ Z e m m o u r a ~}{ }^{1}$, Dehia Nait Ali ${ }^{1}$, MenenaGuemghar ${ }^{1}$, SouhilaChaou $^{1}$, HabibaBaziz $^{1}$, \\ Hocine Remini ${ }^{1}$, Nacer Nait Ali ${ }^{2}$, Lila Boulekbache-Makhlouf ${ }^{1}$ and Khodir Madani ${ }^{1}$ \\ ${ }^{1}$ Université de Bejaia, Algeria \\ ${ }^{2}$ Centre de Recherche Nucléaire d'Alger (CRNA), Algeria
}

\begin{abstract}
Phenolic compounds are a group of aromatic secondary plant metabolites widely spread throughout the plant kingdom. Most of the beneficial characteristics of phenolic compounds have been ascribed to their antioxidant activity. As antioxidants, polyphenols play an important role in the prevention of human pathologies. Moreover, they have many industrial applications, for example they can be used as natural colorants, preservative for foods and applied in the production of paints, paper and cosmetics. Due to these countless beneficial characteristics, researches have been intensified aiming to agricultural and agroindustrial by products as sources of bioactive phenolic compounds. Date stone (Phoenix dactylifera) constitute approximately 10$15 \%$ of the fruit and they are considered a waste by-product of many date processing. In this study, microwave-assisted extraction (MAE) method was investigated for extraction of total phenolic compounds (TPC) from date stone (Phoenix dactylifera) by response surface methodology. The central composite design (CCD) was used to study the effects of three independent variables; microwave power, irradiation time, solvent fraction on the TPC yield and the antioxidant activity. The optimal conditions of extraction allowing to obtain a better yield (efficiency) in TPC are $600 \mathrm{~W}$ for the microwave power, $30 \mathrm{~s}$ for irradiation time and $40 \%(\mathrm{v} / \mathrm{v})$ for ethanol fraction. MAE is more efficient than conventional extraction method to obtain TPC from date stone. The experimental values were reasonably close to the predicted values confirming the validity of the predicted models.
\end{abstract}

\section{Alleviating Obesity and Associated Complications with Supplementation of Phyllanthin, a Lignan from Phyllanthus Species}

\author{
Sneha Jagtap ${ }^{1, \square, ¿, ~ P r a g y a n s h u ~ K h a r e ~}{ }^{3}$, Mahendra Bishnoi ${ }^{3}$, Kanthi Kiran Kondepudi ${ }^{3}$, Shyam Sunder Sharma ${ }^{2}$ and \\ Kamlesh Kumar Bhutani ${ }^{1}$
}

${ }^{1}$ Department of Natural Products, National Institute of Pharmaceutical Education and Research (NIPER), India

${ }^{2}$ Department of Pharmacology and Toxicology, National Institute of Pharmaceutical Education and Research (NIPER), India

${ }^{3}$ National Agri-Food Biotechnology Institute (NABI), India

\begin{abstract}
Plant derived bioactive molecules can provide preventive effects against lifestyle oriented disorders as they are part of daily diet or common supplements. Various plants like Phyllanthus embellica (Indian gooseberry), Phyllanhus niruri, Phyllanthus acidus (country gooseberry) etc. from Phyllanthus species have been part of Asian cuisine and shown to possess medicinal properties. In present study, phyllanthin, one of the major lignan from Phyllanthus species was studied for its effect on high fat diet induced metabolic complications in Swiss mice model. Phyllanthin, at 2 and $4 \mathrm{mg} / \mathrm{kg}$ of body weight along with high fat diet, was supplemented orally over the period of three months. The intervention prevented high fat diet induced changes in anthropometric parameters, serum triglycerides, adipokines, and improved glucose clearance without affecting locomotion and food intake. Phyllanthin supplementation alleviated high fat diet induced secondary inflammation, adipocity, liver fat accumulation and related gene expressions in visceral adipose tissue and liver. Manifestations suggested positive influence of phyllanthin supplementation on chronic diet induced metabolic complications like weight gain, insulin resistance and fatty liver. Present study suggests potential of phyllanthin, a major lignin from Phyllanthus, as nutraceutical against metabolic complications.
\end{abstract}

\section{Development of Peptide Inhibitors against Plasmin by using Phage Display Library}

\author{
Nurdan Ersoz ${ }^{\square, \AA}$ and Fahriye Ceyda Dudak Seker \\ Hacettepe University, Turkey
}

\begin{abstract}
Phage display technique is an ideal tool to select and develop novel enzyme inhibitors with enhanced selectivity. A phage displayed peptide library was used to select novel inhibitors against plasmin enzyme. Plasmin (fibrinolysin) is one of the heat
\end{abstract}


stable milk proteases that can have detrimental effects on the quality of milk. The phage clones having plasmin binding peptides were selected from a commercial M13 phage library and the effect of each phage clone on plasmin activity was determined. Sequence analysis was realized for two peptides showing high inhibitory effect on plasmin and the peptides were synthesized with solid phase peptide synthesis. The sequences had no similarity with natural plasmin inhibitors. Inhibiton mechanisms and kinetics of selected peptides were investigated. The most active peptide showed noncompetitive inhibition with IC50 value of $50 \mu \mathrm{M}$.

\title{
Heavy Metals Contents in Some Spices from Different Origins
}

\author{
Jae-Young Shim ${ }^{\varpi, ¿}$, Yeon Kim, Byung-Min Lee, Ha-Yun Bong, Tae-Yong Cho, Hae-Seong Yoon, Young-Ae Gi and \\ Chang-Hee Lee \\ Center for Food E Drug Analysis, Busan Regional Korea Food and Drug Administration, Republic of Korea
}

\begin{abstract}
A spice is a dried seed, fruit, root bark, or flower of plant or herb used in small quantities for flavor, color, or as a preservative. Globalization has made these spices easily available and increasing their popularity. They can also be contaminated some harmful substance such as trace and heavy metals. It may result in accumulation of these metals in human organs. The aim of this investigation was to estimate and compare among heavy metals ( $\mathrm{Pb}, \mathrm{Cd}, \mathrm{As}, \mathrm{Hg}, \mathrm{Fe}, \mathrm{Cu}$ and $\mathrm{Zn}$ ) contents in available spices from different regions. Samples of spices from 21 different species were purchased from wholesale and internet market in Korea. The concentrations of heavy metals were determined using ultra- microwave and inductively coupled plasma mass spectrometry. Recoveries of the elements analyzed varied between 89.1 103.9\%, and precisions were 0.1 9.8\%. Results were analyzed for statistical significance by analyses of variance using SPSS. This study suggests further monitoring of heavy metals on daily consumption of spices and will contribute to the global overview regarding heavy metals in spices.
\end{abstract}

\section{Evaluation of Extractions Conditions of Quinoa (Chenopodium quinoa Willd.) Protein from a Selected Chilean Ecotype}

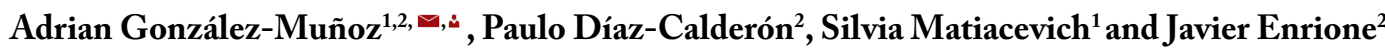 \\ ${ }^{1}$ Universidad de Santiago de Chile, Chile \\ ${ }^{2}$ Universidad de Los Andes, Chile
}

\begin{abstract}
Quinoa is an Andean pseudocereal that has been cultivated for thousands of years. Literature had shown that its high protein content may have beneficial nutritional properties. Nevertheless, the protein content possibly may change in according to the origin of ecotype. The objective of this work was to select a quinoa ecotype with high protein content from five different geographical locations (Bolivia, Chile and Peru) and evaluate processing conditions for protein extraction. Previous work allowed defining that Cahuil ecotype (Chile) has the highest amount of protein (14.8\%) measured by Kjheldal. Four extraction conditions were investigated: time (1-4 hrs), agitation (100-250 rpm), flour-solvent ratio (1:10-1:30 w/v) and $\mathrm{NaOH}$ concentration (30-60 mM). The experiment was carried out in $50 \mathrm{~mL}$ vessels. The suspensions were centrifuged, concentrated by adjusting at $\mathrm{pH} 3.5$ and then neutralized. The soluble protein was determined by Bradford method. The protein yield was calculated as a function of total protein determined by Kjeldhal. $\mathrm{NaOH}$ concentration did not showed to be a significant factor. The protein yield was in accordance with values reported previously on literature. Then aforementioned conditions were scaled-up to $250 \mathrm{~mL}$ flasks and to semi-pilot scale reactor $(5 \mathrm{~L})$ in order to validate the processing conditions, obtaining values of protein yield within $10 \%$ of deviation. In conclusion, it was possible to select conditions for protein extraction from a Chilean quinoa ecotype and then validate these conditions at semi-pilot scale.
\end{abstract}

\section{Contents and Composition of Chlorogenic Acids in Korean Sweet Potato Varieties: Correlation with Their Antioxidative Activities}

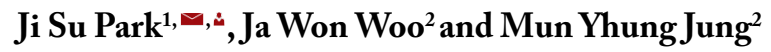 \\ ${ }^{1}$ Korea Food Research Institute, Republic of Korea \\ ${ }^{2}$ Woosuk University, Republic of Korea
}




\title{
Abstract
}

Identification and quantification of the chlorogenic acids in 17 sweet potato varieties were conducted by a high resolution LC-ESI-Q-TOF-MS, LC-MS/MS, and HPLC-DAD analysis. There was a considerable impact of extracting solvent mixture system on the quantity and composition chlorogenic acids. Extracting solvent mixture system greatly influenced the quantity and quality of chlorogenic acids in the extracts of sweet potato. The optimum extracting solvent system was selected for extracting the chlorogenic acids from sweet potatoes. The profiles and quantities of chlorogenic acids in sweet potatoes were greatly dependent with the variety, showing about 30-fold differences in quantity. Juhwangmi contained the highest quantity of Chlorogenic acid (2393.65 mg/g dry weight), while Matnami contained the least quantity of chlorogenic acid (68.62 mg/g dry weight). High correlations ( $>0.97)$ were observed between chlorogenic acid contents and antioxidant potential data from DPPH, ABTS, FARP, and total phenolic assays. Sweet potatoes were statistically classified in 4 well defined groups in terms of their similarity in chlorogenic acid profile and quantity.

\section{Comparison of Mineral Contents in Vegetables (White Cucumber, Red Paprika, Water Parsley and Kohlrabi) Undergoing Different Cooking Methods}

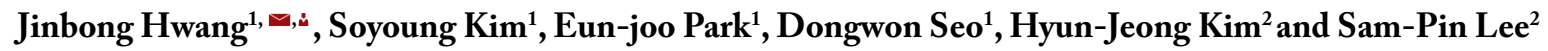 \\ ${ }^{1}$ Korea food research institute, Republic of Korea \\ ${ }^{2}$ Keimyung University, Korea
}

\begin{abstract}
This study attempted to compare minerals contents in vegetables undergoing eight cooking methods such as raw materials, boiling, pan-frying, stir-frying, deep-frying, steaming, roasting and microwave. The vegetable samples, including white cucumber, red paprika, water parsley and kohlrabi were provided by Ministry of Food and Drug Safety (MFDS). The sample was prepared with different cooking methods and then it was digested with $8 \mathrm{~mL}$ of $\mathrm{HNO}_{3}$ and $2 \mathrm{~mL}_{\text {of }} \mathrm{H}_{2} \mathrm{O}_{2}$ using the microwave. This solution was cooled and diluted with deionized water. Diluted solution was injected into the inductively the coupled plasma optical emission spectrometry (ICP-OES) and the inductively coupled plasma mass spectrometer (ICP-MS) to determine minerals ( $\mathrm{Na}, \mathrm{Ca}, \mathrm{K}, \mathrm{P}, \mathrm{Mg}, \mathrm{Fe}, \mathrm{Mn}, \mathrm{Se}, \mathrm{Cu}$ and $\mathrm{Zn}$ ). Most minerals of water parsley showed the highest content among the samples. The highest content was found in the samples cooked by deep-frying. However, the lowest content was in raw water parsley and the other samples cooked by boiling. Potassium for macro-minerals and iron for micro-minerals had the highest content. Hence, it is expected that the mineral contents could be used as nutritional food ingredient database for national health promotion.
\end{abstract}

\section{Simultaneous Qualification and Quantification of Isoflavones in Soybeans and Soybean Products by a HPLC-ESI-TOF-Mass Spectrometry}

\author{
JuYeon Lee ${ }^{\varpi, \varkappa}$, Ji Su Park, Mi Jin Lee and Mun YhungJung \\ Woosuk University, Republic of Korea
}

\begin{abstract}
An analytical method for the rapid isolation, identification, and quantification of isoflavones in soybean and soybean products was developed by a HPLC-ESI-TOF-MS. The isoflavones were identified with the accurate mass data of their respective protonated mass ions, $\mathrm{Na}$ or $\mathrm{K}$ adduct ions, fragment ions, and isotope ion patterns. Extracted ions of the protonated mass ions of the isoflavones were used for the quantifications in soybean products. Twelve different isoflavones in the soybean products were separated, identified and quantified within 12 min separation time. The established HPLC-TOF/MS was an effective analytical method for the characterization and quantification of isoflavones with a short analytical running time, high selectivity, a high linearity, low limit of detection and limit of quantification, high precision, repeatability, and no significant matrix effect. This method requires simple sample preparation procedure of solvent extraction, dilution, and syringe filtration due to the high sensitivity and selectivity.
\end{abstract}




\title{
Functionality of Active Films Based on PCL with Alfa-Tocopherol Loaded in Functionalized Mesoporous Silica
}

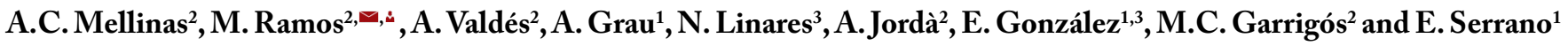 \\ ${ }^{1}$ Laboratorio de Nanotecnología Molecular, Departamento de Quimica Inorgánica, Universidad de Alicante, Spain \\ ${ }^{2}$ Departamento de Quimica Analitica, Nutrición y Bromatología, Universidad de Alicante, Spain \\ ${ }^{3}$ Departamento de Enfermería Comunitaria, Medicina Preventiva y Salud Pública e Historia de La Ciencia, Universidad de Alicante, Spain
}

\begin{abstract}
The opportunity to chemically functionalize the pore surface of siliceous mesostructures with organic molecules constitutes a route for controlling their release by diffusion under specific conditions. This novel approach can be used in the field of active packaging to develop materials able to release antioxidant/antimicrobial compounds, to increase the shelf-life of the packed foodstuff. The aim of this work was to evaluate the release of $\alpha$-tocopherol (TOC) loaded in functionalized mesoporous silica from active PCL films. Samples of MSU-X and MSU-X impregnated with TOC dissolved in ethanol were incorporated into PCL by melt-blending followed by compression molding to obtain films: PCL/MSU-X and PCL/MSU-X-TOC, respectively. Samples of pure PCL, PCL/TOC and PCL/MSU-X/TOC (with direct addition of TOC into PCL) were also obtained. Migration tests were performed at $40{ }^{\circ} \mathrm{C}$ using $50 \%(\mathrm{v} / \mathrm{v})$ ethanol as food simulant at different times, in triplicate. A kinetic study was performed and the amount of TOC released into the food simulant was analyzed by HPLC-UV. Furthermore, the antioxidant activity of TOC released was studied by DPPH and ABTS methods. Finally, antimicrobial activity of the developed films was evaluated by optical density measurements against $E$. coli and $S$. aureus bacteria. Active films containing the functionalized silica-TOC showed a slower release and antioxidant and antimicrobial activity compared to samples containing free TOC. These results suggest a promising approach for the use of mesoporous silica to improve the performance of $\mathrm{PCL} / \mathrm{TOC}$ based films for active packaging by the controlled release of the active agent.
\end{abstract}

\section{Effect of Mineral Salts Addition of Rennet-induced Coagulation of Camel/Cow's Milk on the Rheological Properties and Molecular Structure Determined by Front-Face Fluorescence Spectroscopy}

\section{Mohammad Kamal and Romdhane Karoui ${ }^{\varpi,\llcorner}$}

Université d'Artois, Institut Régional en Agroalimentaire et Biotechnologie Charles Viollette, Equipe Qualité et Sécurité des Aliments (QSA), Unité Ingénierie de Formulation des Aliments et Altérations (IFAA), France

\begin{abstract}
Milk coagulation is the primary step in the development of texture of most dairy products. The present work aims to monitor the rennet-induced gelation at $36{ }^{\circ} \mathrm{C}$ during 45 mins of camel and cow's milk without/with the addition of minerals using dynamic oscillatory rheology and front-face fluorescence spectroscopy. Rheological measurements showed that the final values of storage modulus and loss modulus of camel milk gel were less than those of cow's milk gel. The addition of $\mathrm{CaCl}_{2}$ at 10 and $20 \mathrm{mM}$ to the cow's and camel milks reduced the gelation time, accelerated the enzymatic activity and increased the firmness of the gel. Opposite trend was observed following the addition of $\mathrm{Na}_{2} \mathrm{HPO}_{4}\left(\mathrm{H}_{2} \mathrm{O}\right) 2$ at 10 and $20 \mathrm{mM}$. Regarding the fluorescence spectra obtained after excitation at $290 \mathrm{~nm}$ (emission: 305-420 nm for tryptophan) and emission at $410 \mathrm{~nm}$ (excitation: 252-390 nm for vitamin A), a decrease in the fluorescence intensity, respectively at $375 \mathrm{~nm}$ and 307 and $322 \mathrm{~nm}$ was observed during coagulation, regardless of the type and concentration of salts. The principal component analysis (PCA) applied to the spectral data sets allowed detection of structural changes in casein micelles during coagulation and discriminated between: i) cow's and camel milk, regardless of the added concentration of minerals and ii) cow's/camel milk according to the nature of salt and its concentration. Finally, a high correlation was observed between the data obtained at the macroscopic and molecular levels suggesting the use of fluorescence spectroscopy to derive rheology parameters.
\end{abstract}




\title{
Fast Gas Chromatography-Tandem Mass Spectrometry in Multiple Reaction Monitoring Mode for the Multi Residue Pesticide Analysis in Agricultural Products and Foods
}

\author{
Sol Ji Choi ${ }^{1, \Xi, 2, ~ S u ~ Y e o n ~ P a r k ~}{ }^{1,2}$, Ju Hui Kang ${ }^{1}$,Je Young Shin ${ }^{1}$, Hee Jeong Park ${ }^{1}$ and Mun Yhung Jung ${ }^{1}$ \\ ${ }^{1}$ Woosuk University, Republic of Korea \\ ${ }^{2}$ National Academy of Agricultural Science, Republic of Korea
}

\begin{abstract}
A fast gas chromatography-tandem mass spectrometry in multiple reactions monitoring (MRM) mode was successfully established for the multi residue pesticide analysis (160 pesticides) in agricultural products and foods. The column used was a nonpolar capillary column (5\% phenyl 95\% methyl siloxane, $30 \mathrm{~m}$ x $0.25 \mathrm{um}$ ). Helium was used as a carrier gas. Full separation and identification of the 160 pesticides were achieved within 23 mins of gas chromatography running time. The single mass spectrometry with full scan mode and tandem mass spectrometry with product ion scan mode were conducted to identify the transitions for the optimum quantification sensitivity of pesticides. This established analytical method showed the very low LOD for the pesticides, ranging from 0.02 to $10 \mathrm{ug} / \mathrm{L}$. The calibration curves obtained by this method showed excellent linearity $\left(r^{2}=0.994-1.000\right)$ of most of the tested pesticides with a few exception $\left(r^{2}=0.983\right)$ in an established concentration range. High recovery rates with the known amount spiked samples were obtained, indicating the high accuracy. The accuracy of the method was further confirmed by participating Fapas international proficiency test program. This method could be successfully applied to analyze the multi residue pesticides in various agricultural and foods.
\end{abstract}

\section{Cyclodextrin-Based Nanosponges: A Versatile Vehicle Exploitable for Foodstuff Fortification}

\author{
Vito Coviello ${ }^{1, ๘, \varkappa}$, Stefania Sartini ${ }^{1}$, Maria Cristina Gamberini ${ }^{2}$, Cecilia Baraldi ${ }^{2}$ and Concettina La Motta ${ }^{1}$ \\ ${ }^{1}$ Dipartimento di Farmacia, Università di Pisa, Italy \\ ${ }^{2}$ Dipartimento di Scienze della Vita, Università degli Studi di Modena e Reggio Emilia, Italy
}

\begin{abstract}
Nano-sized colloidal carriers have been recently proposed as innovative systems exploitable for small molecules delivery, as they allow a targeted and prolonged release of poorly water-soluble compounds, improving their bioavailability and modifying their pharmacokinetic parameters while preserving their stability. Accordingly, they represent an intriguing vehicle which can be exploited to distribute suitable supplements within foodstuff for their fortification. Among the known colloidal carriers, nanosponges obtained from beta-cyclodextrins represent the most versatile ones. These are solid, porous and bio-compatible polymers, prepared through condensation polymerization reaction between beta-cyclodextrin and a suitable cross-linking agent, often represented by diphenyl carbonate. The resulting nano-particulate, three-dimensional structure is able to form inclusion complexes with different types of both lipophilic and hydrophilic drugs, which can be thus formulated as oral, parental, and topical dosage forms. Here, we present a new delivery system, innovative, versatile and fully biocompatible, which proved to host a novel prototype of food supplement, DB103, increasing its solubility, preserving its stability and allowing its delivery. Novel inclusion complex was characterized through physical and chemical data, exploiting UV and IR analyses, differential scanning calorimetry (DSC), scanning electron microscopy (SEM) and Raman studies. Loading efficiency and in vitro dissolution studies were performed as well, to demonstrate the ability of the novel nanosponge to release the target drug slowly and continuously, thus confirming its soundness as a novel sustained drug-delivery system.
\end{abstract}




\section{Submit Your Next Manuscript}

We are currently accepting submissions from new authors. Read our latest issue articles and submit your next manuscript to Journal of Food Chemistry \& Nanotechnology (JFCN).

Submit Online: http://www.editorialmanager.com/usg

Citation: Proceedings of the First International Conference on Food Chemistry \& Technology. J Food Chem Nanotechnol 2(Supp1 2): S1-S45.

Copyright: This is an Open Access article distributed under the terms of the Creative Commons Attribution 4.0 International License (CC-BY) (http://creativecommons.org/licenses/by/4.0/) which permits commercial use, including reproduction, adaptation, and distribution of the article provided the original author and source are credited. Published by United Scientific Group.

Received: November 30, 2015 Accepted: March 4, 2016 Published: March 10, 2016 